\title{
Holism as the empirical significance of symmetries
}

\author{
Henrique Gomes ${ }^{1}$ (1D
}

Received: 31 October 2019 / Accepted: 6 July 2021 / Published online: 13 August 2021

(C) The Author(s) 2021

\begin{abstract}
Not all symmetries are on a par. For instance, within Newtonian mechanics, we seem to have a good grasp on the empirical significance of boosts, by applying it to subsystems. This is exemplified by the thought experiment known as Galileo's ship: the inertial state of motion of a ship is immaterial to how events unfold in the cabin, but is registered in the values of relational quantities such as the distance and velocity of the ship relative to the shore. But the significance of gauge symmetries seems less clear. For example, can gauge transformations in Yang-Mills theory-taken as mere descriptive redundancy-exhibit a similar relational empirical significance as the boosts of Galileo's ship? This question has been debated in the last fifteen years in philosophy of physics. I will argue that the answer is 'yes', but only for a finite subset of gauge transformations, and under special conditions. Under those conditions, we can mathematically identify empirical significance with a failure of supervenience: the state of the Universe is not uniquely determined by the intrinsic state of its isolated subsystems. Empirical significance is therefore encoded in those relations between subsystems that stand apart from their intrinsic states.
\end{abstract}

Keywords Gauge Theory · Direct empirical significance · Holism · Fields · Separability

\section{Introduction}

\subsection{Overview of the debate and my position within it}

In its broadest terms, a symmetry is a transformation of a system which preserves the values of a relevant (usually large) set of physical quantities. Of course, this broad idea is made precise in various different ways: for example as a map in the space of states, or on the set of quantities; and as a map that must respect the system's dynamics, e.g. by mapping solutions to solutions or even by preserving the value of

In honour of G. t' Hooft's 20th year Nobel prize celebration.

Henrique Gomes

gomes.ha@gmail.com

1 Trinity College, University of Cambridge, Cambridge, CB2 1TQ, UK 
the Lagrangian functional on the states.

The broad idea is also associated with various debates. ${ }^{1}$ For example, should we say that a symmetry transformation applied to the whole universe cannot yield a different physical state of affairs? And relatedly: should we prefer a reduced i.e. quotiented formalism, so that if presented with a state space $\mathcal{S}$ partitioned into the orbits of a group of symmetries $\mathcal{G}$ acting on $\mathcal{S}$, we prefer the reduced state space whose elements are the orbits, i.e. $[s] \in \mathcal{S} / \sim$ ? (where $s \sim s^{\prime}\left(s, s^{\prime} \in \mathcal{S}\right.$ ) means that $s$ and $s^{\prime}$ are related by a symmetry transformation: $s^{\prime}=\xi \cdot s$, for $\xi \in \mathcal{G}$ and $\cdot$ some action of $\mathcal{G}$ on $\mathcal{S}$, and square brackets denote an entire equivalence class).

These "defining features" of symmetries are of central concern for one recent philosophical debate. More specifically, the debate is about whether gauge symmetries can have a direct empirical significance. Of course, all hands agree that symmetries have various important empirical implications. The obvious examples come from the Noether theorems: the restrictions on the equations of motion entailed by Noether's second theorem, and the (approximately) conserved charges given by Noether's first theorem. In other words, symmetries imply the (extraordinary) fact that charges are conserved. ${ }^{2}$

But some familiar symmetries of the whole Universe, such as velocity boosts in classical or relativistic mechanics (Galilean or Lorentz transformations), have a direct empirical significance when applied solely to subsystems. Thus Galileo's famous thought-experiment about the ship — that a process involving some set of relevant physical quantities in the cabin below decks proceeds in exactly the same way whether or not the ship is moving uniformly relative to the shore-shows that sub-system boosts have a direct, albeit relational, empirical significance. For though the inertial state of motion of the ship is undetectable to experimenters confined to the cabin, yet the entire system, composed of ship and sea registers the difference between two such motions, namely in the different relative velocities of the ship to the water. ${ }^{3}$

So the question arises: Can other symmetries-especially gauge symmetrieshave a similar direct empirical significance when applied to subsystems?

\footnotetext{
${ }^{1}$ See the essays in Brading and Castellani (2003) and the references therein.

${ }^{2}$ Accordingly, in this debate, such familiar implications are often called the 'indirect' empirical significance of symmetries (IES); and through them, symmetries carry immense explanatory power.

${ }^{3}$ As often is the case in physics, the characterization of direct empirical significance used here may rely on certain approximations. For surely, with the right equipment (such as a window), the person within the cabin could discern movement of the ship from within, and different movements of the ship could create different sorts of eddies and turbulence in the sea. This sort of idealization is ubiquitous in physics, and generally unproblematic. This is echoed in Greaves \& Wallace (2014, p. 52, footnote 7): “'Dynamical isolation' is of course approximate in practice, as no proper subsystem can in practice be perfectly isolated. It is also relative to the observation capabilities of relevant observers (if Galileo had included a GPS tracker in his list of the cabin's accoutrements, things would have been rather different)." Nonetheless, approximate symmetries have consequences for theory construction (cf. Earman (2019) and also Pitts (2011), and Fletcher (2021) for a formal treatment of approximate symmetries through similarity relations). As a side note, from now on, I will prefer "sea" to "shore"; this restriction eliminates the need to discuss translations in addition to boosts (Maudlin, 1993), and places the two subsystems in direct contact, as in the case we will explore.
} 
For gauge symmetries are normally taken to encode descriptive redundancy: a view I will endorse. That is, they arise in a formalism that uses more variables than there are physical degrees of freedom in the dynamical system described.

This descriptive redundancy means that the natural answer to our question is 'No'. For surely, while a "freedom to redescribe" may have some indirect empirical implications, ${ }^{4}$ it could not have the content needed for a direct empirical significance, like the one illustrated by Galileo's ship. This 'No' answer was developed in detail by Brading and Brown (2004) in response to various discussions such as (Kosso, 2000). They take themselves-I think rightly, in this respect-to be articulating the traditional or orthodox answer.

The 'Yes' answer has been argued for by Greaves and Wallace (2014), building on Healey (2009). I will agree with some aspects of both Brading and Brown (2004)'s and Greaves and Wallace (2014)'s analysis of symmetries. But, unlike either of them, I will recast the topic to focus on gauge-invariant information about-i.e. states of-regions. My own conclusion will be that only a finite subset of gaugetransformations, usually called 'global' (but here called 'rigid', cf. Section 2.2), can have direct empirical significance, or DES, as it is known in the literature (an abbreviation I will adopt).

We have glossed the heuristic meaning of DES, and I will provide more precise definitions in later sections, but an informal sketch is as follows: First, the broad notion of DES is a matter of the existence of transformations of the universe (or of the models of the theory) possessing the following two properties (articulated in this way by Brading and Brown (2004)):

(i) the transformation leads to an empirically different scenario, and

(ii) the transformation is a symmetry of the subsystem in question (e.g. Galileo's ship).

If such transformations exist, the symmetries of the theory-related to DES through (ii) - are said to exhibit DES. The empirical significance is to be witnessed by observers that lie outside the subsystem - it cannot be detected by those confined within it. Therefore, DES combines an inside and an outside perspective and, in this limited sense, acquires an epistemic dimension, or at least one that considers physical information as it is intrinsically accessible within a subsystem.

Brading and Brown (2004) argue that gauge symmetries cannot exhibit DES according to (i) and (ii), while Greaves and Wallace (2014) argue that they can. I myself will argue for a 'Yes' answer, but will approach the question in terms of gauge-invariant information.

By thus proceeding in terms of gauge-invariant information, I will identify DES as defined by (i) and (ii) above with a particular type of failure of Global Supervenience on Subsystems (GSS): ${ }^{5}$ this failure is a form of holism-hence my title.

\footnotetext{
${ }^{4}$ By arriving at a local description to redescribe by weakening the global symmetries of the theory, the conserved charges implied by the global symmetries are required to couple to fields in such a way that conservation laws are dynamically respected (this is the content of e.g. the Gauss law).

${ }^{5}$ In the context of gauge systems under study here, a failure of global supervenience on subsystems, is close in spirit to Myrvold's global patchy non-separability (Myrvold, 2010), which he articulated for the
} 
I will show that relational DES occurs only when the gauge-invariant global state fails to supervene on the collection of intrinsic, gauge-invariant local states of the components of some arbitrary partition (of space or spacetime).

When environment and subsystems are on a par, DES can be rephrased as a matter of global supervenience. GSS is upheld-indicating the absence of DES-when the intrinsic physical states of those subsystems composing the whole uniquely determine the physical state of the whole; the data contained in the intrinsic subsystem states need not be augmented by relational data in order to uniquely specify the state of the joined system. When GSS fails-indicating the presence of DES-there can either be many physical states of the whole which are formed from the same physical states of the individual subsystems, in which case one is missing some relational information, or there can be no valid states of the whole, in which case the subsystems states are incompatible.

Here I should make it clear that I am not claiming ontological priority for the subsystems composing the whole. Subsystems don't exist "before" the whole. The division of the Universe into subsystems is not mandatory, but it appears in item (ii): so we must consider what physical information is intrinsic to a subsystem when evaluating the direct empirical significance of symmetries.

It is also important to flag, right at the beginning, a related confusion that permeates the debates on DES: for both DES (direct empirical significance of subsystem symmetries) and GSS (global supervenience on subsystems) are about the composition of subsystem into a larger system, not about the decomposition of a larger system into subsystems. To be clear, in this paper, regarding the less important decomposition, I will only countenance the case where a given physically allowed state of the universe decomposes into physically allowed states of its subsystems. I know of no examples violating this assumption. Therefore, we will focus on the more interesting of the two cases of failure of GSS: namely, the one where, given just the intrinsic physical states of the subsystems, there are physically distinct possibilities to join these states into some physical state of the Universe. That is, the relation between states of the Universe and states of its subsystems are many-to-one, because there is relevant relational information that cannot be registered intrinsically within each subsystem. In these cases, we will say there is Global Non-Supervenience on Subsystems and denote it by $\neg$ GSS. When $\neg$ GSS prevails, the states of the whole are not fully encoded in either subsystem: it is encoded in the relations between the two subsystems.

In certain situations, such as in Galileo's ship, and, as I will argue, in gauge theory, there is remarkable order to this variety of physical states of the whole, an order also encoded in the structure of $I$. Namely, each element of this variety can be transformed into another by a subsystem symmetry which does not extend beyond the boundary of the subsystem. In other words, $I$ carries the structure of finite-dimensional symmetry group of the subsystem. For Galileo's ship, these are the Galilean transformations;

holonomy approach to gauge theories. But I refrain from adopting this nomenclature because (i) I do not focus on holonomies, and (ii) it does not apply to finite-dimensional systems like Galileo's ship. I will briefly comment again on this relation in footnote 38 in Section 5.3. 
and for the gauge theory, we will see that they are (sub)groups of the Lie group characterizing the theory. In this manner, DES becomes a matter of $\neg$ GSS.

In sum, technicalities apart, my main claim is that both Galilean boost symmetry for particle systems and gauge symmetry for certain field theories carry Direct Empirical Significance (DES) through a failure of Global Supervenience on Subsystems ( $\neg$ GSS). This holism is empirically significant, since it registers physical-i.e. gauge-invariant—-differences in the entire system and we take such differences to lead to empirically distinguishable universes. Moreover, the implied under-determination of the physical state of the whole universe by the physical state of its subsystems is encoded in a subsystem symmetry, but only as seen from the 'outside perspective'; again in accord with the above construal of DES.

This last paragraph summarizes my construal of DES in this paper. To flesh it out, we first need to revisit a different debate in the philosophy of gauge theory, which I describe in the next section.

\subsection{External sophistication}

If the standard notion of DES in (i) and (ii) is to be identified with a failure of GSS, we first need to develop a physically meaningful notion of composition of those subsystems that possess descriptive redundancy.

This requirement leads us to revisit one other important debate in the philosophy of gauge, already mentioned at the start of the previous section. Namely, given a theory whose set of universe-descriptions - 'states'-is partitioned by a group of symmetries, we can take one of two attitudes:

(a) Reduction:- try to write down a reduced theory whose states correspond to the cells of the partition; or

(b) Sophistication about symmetries ${ }^{6}$ :- resist quotienting the given theory, but take two symmetry-related states to be isomorphic.

I'll advocate a third position (c), which applies only in the presence of subsystems. This position allows reduction for the entire universe, but not for its subsystems. Thus the position is compatible with the criterion of physical discernibility of the theory in question: that empirically discerns two different states $s_{1}$ and $s_{2}$ of the universe if and only if $\left[s_{1}\right] \neq\left[s_{2}\right]$.

But for subsystems, the question is more subtle, for there are two perspectives we can take: one from the inside, or intrinsic; and one from the outside, or extrinsic,

\footnotetext{
${ }^{6}$ Sophistication has long been advocated for diffeomorphisms and spacetime metrics (see Pooley (2013) and references therein). The nomenclature was originally used for sophisticated substantivalism: points of spacetime may have identity, but this identity comes only through the complex web of inter-relations between different fields of the theory, and is in this way entirely dependent on the state. Some general features of this position have more recently also been suggested for gauge theories (Dewar, 2017; Gomes, 2019; Caulton, 2015). Dewar describes it thus: "Whereas a reduced theory converts a class of symmetryrelated models into a single model, sophistication converts a class of symmetry-related models into a class of isomorphic models." Some philosophers (e.g. Healey 2007, Sec. 4.2 and Maudlin (1998)) have resisted the analogy between the descriptive redundancy of the metric in gravitational theories and of the connection in gauge theories. Pace these philosophers, I see no reason for their resistance, but that is a topic for another day.
} 
in accord with the definition of DES. If we are interested in discriminating between (intrinsically) distinct physical possibilities, then surely those states which cannot be (intrinsically) discriminated are to be counted as one, and so, to that end, 'reduction' would still be acceptable.

But, when we combine the subsystem with the rest of the world, we are required to exploit subsystem symmetries in a real physical sense: as emphasized first by Rovelli (2014), reduced representations of subsystems cannot be straightforwardly coupled to each other. For coupling, we need to keep gauge-variant elements in the theory.

In Gomes (2019), it was similarly argued that reduction should only be endorsed for the entire universe: coupling regional states may require a re-expression of the states as particular gauge representatives of the physical states $;{ }^{7}$ and thus, for regions, gauge-information should not be entirely eliminated.

Therefore, I have argued that we should delineate a third attitude (Gomes, 2019):

(c) No external reduction of subsystem symmetries:-Fix unique representations of the intrinsic physical states of the subsystems (i.e. from the internal perspective), but then allow these representations flexibility from an external perspective, as e.g. required for the smooth coupling of the states of subsystems. Reduction is a more strict attitude towards symmetries than sophistication, but option (c) should be construed as permissive: it encompasses an attitude of both internal and external sophistication, but it cannot encompass one that admits both internal and external reduction.

Take the example used in Rovelli (2014): a non-relativistic classical system of $\mathrm{N}$ particles with translational invariance. From the intrinsic perspective of the subsystem, one could eliminate redundancy by taking the inter-particle distances as a new, autonomous set of coordinates, but this would leave no 'handle' for other subsystems to couple to. The joint system of autonomous coordinates for two such sets of particles (say $N_{1}$ 'red' and $N_{2}$ 'green') cannot express different ways of composing the subsystems - whether the center of mass of the 'reds' are five or ten feet away from the center of mass of the 'greens' along some direction does not register in these variables. From a degree of freedom count, we have clearly gone overboard: we have eliminated six degrees of freedom of the joint system - the position of the center of mass of 'reds' (three degrees of freedom) and the position of center of mass of 'greens' (three degrees of freedom)—when only three were eliminable: the position of center of mass of $\{$ reds $\} \cup\{$ greens $\}$ ).

On the other hand, fixing the isolated subsystem's coordinates by reference to its center of mass, while leaving the center of mass embedded in Euclidean space, still affords us enough flexibility to characterize both the subsystems intrinsic degrees of

\footnotetext{
${ }^{7}$ Rovelli focused on the coupling between different types of fields, or, in the finite-dimensional case, on the coupling of two different particle systems (Rovelli, 2014). In Gomes (2019) I extended that requirement to the coupling of fields in regions. See also Dougherty (2017) for a 'stack-theoretic' argument emphasizing the problems of reduction for the coupling of regions: his notion of separability requires the preservation of gauge-related representations, to be kept as isomorphic but not identified. That is, I construe Dougherty's view as a defense of position (b) motivated by the composition of subsystems (but using stack-theory). Also (implicitly) using a stack-theoretic approach, Nguyen et al. (2018) emphasize that gauge transformations are not just re-descriptions, but also parameterize the different ways in which regional field spaces can be composed into global field spaces.
} 
freedom and a rigid subsystem translation with respect to another subsystem. This is a very simple example of a "covariant gauge-fixing", and in practice, it is how we implement option (c).

In my analysis of DES in the context of holism, the flexibility allowed by option (c), 'External sophistication' for short, is employed for melding the subsystems' physical content into the physical content of the joint state. ${ }^{8}$ Option (c) allows us to have our cake and eat it too: we can both parametrize the intrinsic physical possibilities of the subsystems in a one-to-one manner, and yet keep track of those degrees of freedom that would be redundant from the intrinsic point of view but which must be retained, to be pressed into service for composing subsystems (Gomes, 2019).

\section{Direct empirical significance}

I start, in Section 2.1, by construing DES in terms of properties of transformations of the Universe. This description of DES immediately runs into some cumbersome notation when applied to gauge theories. Therefore, in Section 2.2 I introduce a new terminology which better distinguishes the relevant categories of transformations. Then, having got the right nomenclature for addressing DES in the context of gauge theories, in Section $2.3 \mathrm{I}$ apply it to re-express the debate in these better terms. In Section 2.4, I then proceed to offer an appetizer of my criticism of Brading and Brown (2004) and Greaves and Wallace (2014)'s construals of DES, and supplant those construals with my own. Thus here I describe the relation between $\neg$ GSS and DES.

\subsection{DES as a transformation of the universe}

Brading and Brown (2004) frame the definition of DES in terms of two conditions. First, a transformation cannot be a symmetry of the entire universe, otherwise it would not have any direct empirical significance. But second, it needs to act as a symmetry for subsystems, otherwise the transformation in question could hardly be called a symmetry. Thus Brading and Brown define:

Definition 1 (Direct Empirical Significance (DES) as a transformation) A symmetry has direct empirical significance if it is specified by a transformation that satisfies the following conditions: ${ }^{9}$

\footnotetext{
${ }^{8}$ Nguyen et al. (2018) accept something like position (c). The main difference is that they don't impose a unique representative on regions (they just attach the entire groupoid of fields to each region). In other words, they are not interested in uniquely and explicitly parametrizing the physical content of each region, employing instead a "stack-theoretic" picture.

${ }^{9}$ Both Teh (2016) and Greaves and Wallace (2014) add a condition of dynamical isolation between the two subsystems. Teh takes this to justify an asymptotic treatment for the subsystem in question. We won't need to make this isolation condition explicit: it emerges from the criteria.
} 
1. Transformation Condition: the transformation must yield an empirically different scenario. In our words: the transformation in question is not a symmetry of the world as a whole.

2. Subsystem Symmetry Condition: The evolution of the untransformed and transformed subsystems must be empirically indistinguishable from the interior point of view. In our words: the transformation should count as a symmetry when restricted to the subsystems composing the entire system. This is the subsystem symmetry with DES. ${ }^{10}$

Thus in the example of Galileo's ship, the entire system-both ship and sea-is in different states if the ship is heading through calm waters towards the North-West at $10 \mathrm{~km} / \mathrm{h}$ or towards the South at $20 \mathrm{~km} / \mathrm{h}$. The entire system thus satisfies the first condition (Transformation). Nonetheless, inside the cabin, you would not be able to distinguish the two scenarios (cf. footnote 3): so the subsystem satisfies the second condition.

As is clear from the above, the empirical significance cannot be witnessed by observers within the subsystem and, in that way, DES combines an inside and an outside perspective: inside it considers intrinsic physical information that is accessible within the subsystem; outside it considers an overall change in the state of the universe.

\subsection{Two distinctions}

At this point in the discussion, standard terminology gets in the way of clarity. When used in conjunction with subsystem-Universe distinctions, the words 'local' and 'global' acquire other possible meanings, and may pull intuition in different directions (see p. 648 of Brading and Brown (2004)). Therefore, it is useful to introduce a nomenclature that distinguishes these meanings. Therefore I will take the following to apply to sets of transformations:

\footnotetext{
${ }^{10}$ Although Brading and Brown (2004) distinguish a subsystem and its environment, and thus have only the singular 'subsystem' in their definition, it is customary to focus on relational DES, i.e. DES with the environment taken on a par with the subsystem in question. Thereby, the environment is taken as just one more subsystem, and transformations of the environment are to be considered just as much as transformations of the subsystem. In particular, this demotion of the environment to subsystem status means that one excludes the exclusively external relations of the environment from its state. A non-relational definition of DES would not require condition 2 to apply to the environment (cf. footnote 14), that is, it would include transformations that are not symmetries of the intrinsic state of the environment. The standard argument against non-relational DES is that only relational DES has a principled connection between a subsystem symmetry and physically distinct universes. To glean the difficulty with non-relational DES, consider the following example: in the Galilieo ship scenario, imagine a transformation that arbitrarily changes the configuration of the interior of the beach-taken as part of the environment-but otherwise keeps the subsystem-intrinsic and all other relational information untouched. If definition 1 did not require the physical state of the environment to be preserved, such a transformation would be included and therefore correspond to a symmetry with DES. But an arbitrarily changing beach has little to do with symmetries. See also footnote 25 .
} 
- Universal: A set of universal transformations has elements that act on the world as a whole. The set of universal transformations may depend on an infinite or finite number of parameters.

- Regional: A set of regional transformations has elements that need only apply to a subsystem of the world. ${ }^{11}$ The set of regional symmetry transformations may also depend on an infinite or finite number of paramaters.

- Malleable (aka 'local'): A set of malleable transformations depends on an infinite number of parameters: e.g. each element is specified by an arbitrary smooth function over a given manifold or region of a manifold. Here, the usual label is 'local'. But using 'local' invites confusion with the above category, 'regional'. I will therefore prefer the term 'malleable'. A set of malleable symmetry transformations can be either regional or universal.

- Rigid (aka 'global'): A set of 'rigid' symmetry transformations depends only on a finite number of parameters. This is to be contrasted with malleable. The usual label is 'global'. But again, this term invites confusion, namely with the above category, 'universal'. So I will prefer the term 'rigid'. A set of rigid symmetry transformations can be either regional or universal.

Therefore a set of symmetry transformations may lie in any of the following four combinations of the above categories: regional and rigid, regional and malleable, universal and malleable, or universal and rigid. ${ }^{12}$ There is a possible inclusion along both axes of the distinction: a set of rigid transformations can, but need not be, embedded in a set of larger, malleable transformations; and similarly a set of regional transformations can be embedded in a set of universal transformations. Here we will label a given set by the most restrictive combination of categories to which it belongs.

Regional transformations are under-studied in the physics literature, but are known to hide many surprises: see e.g. Regge and Teitelboim (1974), Balachandran et al. (1996), Donnelly and Freidel (2016), Gomes et al. (2019), and Gomes (2019). As to universal transformations, the rigid ones are familiar; they are associated with the standard treatments of Noether's first theorem, and thus correspond to conserved charges (Noether, 1917; Kosmann-Schwarzbach \& Schwarzbach, 2011; Brading \& Brown, 2000; Butterfield, 2007). The malleable universal transformations are associated to constraints, or relations between the equations of motion (such as the Hamiltonian constraints or the Bianchi identities of general relativity and the Gauss constraint in electromagnetism). ${ }^{13}$

\footnotetext{
${ }^{11}$ As in our discussion hitherto, this is often called a 'subsystem symmetry' (Greaves \& Wallace, 2014; Teh, 2016), but here I employ this alternative nomenclature because my interest will be solely in subsystems formed by restricting to a spacetime region.

${ }^{12}$ Teh (2016) labels the transformations with underlining: as local (meaning regional), and local (meaning malleable), and global (meaning universal), and global (meaning rigid); but I feel this underlining also invites confusion.

${ }^{13}$ In the Hamiltonian treatment, the symmetries are represented as flows in the constrained phase space, with orbits being the manifold to which the (integrable) flows are tangent. In the Lagrangian treatment, symmetries are represented as orbits in configuration space (Lee \& Wald, 1990). A powerful formalism which lies in between the Hamiltonian and Lagrangian is the covariant symplectic formalism (Lee \& Wald, 1990; Crnkovic \& Witten, 1987). It is most useful in discussing canonical (or Hamiltonian) features of a system while retaining easy access to spacetime covariance.
} 


\subsection{The debate re-expressed}

Using this nomenclature, we can re-express Definition 1 and better address the subtleties of applying it to gauge symmetry. Thus Definition 1 says that DES arises if there are transformations that are not universal symmetries and yet whose restrictions are regional symmetries. The question is which, if any, regional malleable symmetry can be obtained in this way, and thus be awarded DES.

Finite-dimensional theories, i.e. ones which do not involve fields, generally only have rigid symmetries (such as translations, etc.). In those cases, the strictly regional symmetries can give different values for appropriate physical quantities, viz. relational quantities relating the transformed subsystem to the rest of the universe. This is of course what Galileo's ship illustrates. In this case, the clear distinction between universal and regional rigid symmetries is illustrated in an uncontroversial case of DES.

But the situation for malleable symmetries seems different. In certain examples, the generators of malleable symmetries are spacetime vector fields; in others, they are Lie-algebra-valued scalar fields, acting on an internal space over each spacetime point. In any case, it is easy to imagine a malleable symmetry acting on a region of spacetime and not on another. In this case, the malleable transformation should smoothly tend to the identity at the boundary between the regions, lest it create discontinuities in the fields. But then it seems we could suitably extend any such regional malleable symmetry to the rest of the Universe simply by extending the identity transformation from the boundary to the rest of the Universe. The conjunction of the two regional transformations-one that tends to the identity at the boundary and the other the identity on the rest of the universe-would be a universal (malleable) symmetry, and thus could not have DES, as it would fail to satisfy condition 1 - (Transformation $)$ of Definition 1.

According to Brading and Brown (2004), this is precisely the case:

"Thus, a transformation applied to one subsystem will involve the other subsystem, even if only because the transformation of the gauge field goes smoothly to the identity. In conclusion, there can be no analogue of the Galilean ship experiment for local gauge transformations, and therefore local gauge symmetry has only indirect empirical significance (being a property of the equations of motion)." (p. 657)

Greaves and Wallace (2014) articulate DES for gauge theory in a manner that fosters DES for gauge symmetries. They focus on subsystems as given by regions, and thereby identify transformations possessing properties 1 and 2 of Definition 1 by first formulating the putative effects of such transformations on the gauge fields in these regions.

In particular, they focus their attention on relational DES. That means they consider the environment to be on a par with the subsystem in question (cf. footnote 10). In this case the transformation in Definition 1 must obey property 2-it must also 
be a symmetry of the environment of the subsystem. ${ }^{14}$ And thus we can diagnose DES as originating in the relations between the subsystems (and, ultimately, as I will show, in a failure of supervenience of the global state on the intrinsic states of the subsystems).

More precisely, for a given subsystem state $s$, they claim the relational DES transformations are in 1-1 correspondence with the following quotient between two groups of transformations:

$$
\mathcal{G}_{\mathrm{DES}}^{\mathrm{GW}}(s) \simeq \mathcal{G}(s \mid \partial) / \mathcal{G}_{\mathrm{Id}},
$$

where $\mathcal{G}\left(s_{\mid \partial}\right)$ are the gauge transformations of the region which preserve the state $s$ at the boundary of the region, and $\mathcal{G}_{\text {Id }}$ are the gauge transformations of the region which are the identity at the boundary. Here the equivalence class between two transformations $\xi, \xi^{\prime} \in \mathcal{G}\left(s_{\mid \partial}\right)$ is taken as $\xi \sim \xi^{\prime}$ iff $\xi^{\prime}=\eta \xi$ for some $\eta \in \mathcal{G}_{\text {Id }}{ }^{15}$ The rough idea is that even if certain transformations would not preserve all possible states at the boundary, they will preserve some of those states. ${ }^{16}$ So, $\mathcal{G}_{\text {Id }}$ is a subgroup of $\mathcal{G}\left(s_{\mid \partial}\right)$, and one would like to 'factorize out' from those transformations those that would preserve all states at the boundary (i.e. the boundary-identity transformations): so one defines the quotient group $\mathcal{G}(s \mid \partial) / \mathcal{G}_{\text {Id }}$.

Several assumptions go into the results of Greaves and Wallace (2014) and Brading and Brown (2004), and we will challenge some of these once we have described our own results (see Section 4).

\subsection{My own position in the debate: rigid variety}

Overall, I will argue for a position not considered by either the Greaves and Wallace (2014) or the Brading and Brown (2004) camp: an appropriate selection of rigid regional symmetries_-but not all the malleable ones! - can retain direct empirical significance (DES) in both the finite-dimensional case and in the field-theoretic case. In very specific circumstances, and according to a precise method, the rigid symmetries will be identified among the malleable ones-they are the ones that leave the gauge potential invariant but which shift the matter fields, and they will be the only

\footnotetext{
${ }^{14}$ While Greaves and Wallace (2014) do allow for the larger, non-strictly relational quotient group-of all subsystem symmetries quotiented by the interior ones, where condition 2 need not apply to the environment, or the complement of the subsystem - they do not investigate this overly general definition of DES. For, in their nomenclature, there could be no 'principled connection' between an element of the wider group and empirical significance (Greaves \& Wallace, 2014, p.86,87). See footnotes 10 and 25 here for more on the 'principled connection' and the treatment of the environment as solely a reference and not a subsystem; and Ramirez and Teh (2019) for one possible interpretation of the term 'principled connection' for non-relational DES.

${ }^{15}$ A group is just a set closed under an associative invertible binary operation. That is, if $\xi$, $\xi^{\prime} \in \mathcal{G}$, then $\xi \xi^{\prime} \in \mathcal{G},\left(\xi \xi^{\prime}\right) \xi^{\prime \prime}=\xi\left(\xi^{\prime} \xi^{\prime \prime}\right)$ and for all $\xi \in \mathcal{G}$ there exists a $\xi^{-1}$ such that $\xi^{-1} \xi=$ Id, where $\xi \operatorname{Id}=\xi$ (here we will not need to distinguish left and right inverses). The quotient is well-defined for normal subgroups: namely, $\mathcal{G} / \mathcal{H}$ is well-defined as a group if given $\eta \in \mathcal{H}, \xi \eta \xi^{-1} \in \mathcal{H}$ for all $\xi \in \mathcal{G}$. This holds in the example above.

${ }^{16}$ One could think of it as follows: some state $s$ might be 'periodic' in what it says about the boundary; so that a transformation with the same period at the boundary will fix the state $s$ at the boundary even though the transformation is not the identity there (i.e does not fix all states $s^{\prime}$ at the boundary. That is: when the transformation is restricted to boundary, it does not fix each state thus restricted to boundary).
} 
ones lying in the kernel of a (configuration-dependent, in the non-Abelian case) elliptic differential operator-and they will be transformations with DES as per Definition 1.

Rigid variety and relational DES In the following, to make matters concrete, the fieldtheories I will focus on are general classical Yang-Mills theories in the presence of matter. The spacetime fields in question will be the standard, smooth gauge potentials, $A$, and charged scalars, $\psi$, valued in the appropriate vector spaces, which I discuss in more detail below, in Section 3.1. I denote the doublet of these two fields by: $\varphi=(A, \psi)$, and the space of such doublets by $\Phi \ni \varphi$. The subsystems will consist of regions in the manifold, to which the fields get restricted. Thus the subsystems in questions are regional, and thus we refer to Global Supervenience on Regions (to avoid unnecessarily detailed acronyms, and since R(egions) are certain types of S(ubsystems), we will denote these also by the more inclusive GSS). This initial setup is standard in the debate about the DES of gauge symmetries, and is applicable to all of the approaches considered here.

DES combines an inside and an outside perspective: inside it considers physical information that is intrinsically accessible to subsystems; outside it considers an overall change in the state of the universe. Definition 1 can be formulated as a failure of GSS because its requirement 2 is about information that is intrinsically available to a subsystem. Therefore, for relational DES, a transformation that does not change the intrinsic physical states of its subsystems and yet changes the physical state of the Universe must be changing the relations between the subsystems. Therefore, for DES to exist, there must be a physical variety of universes which are made up from the same physical states of the subsystems.

Accordingly, within this context of Yang-Mills fields, I define: ${ }^{17}$

Definition 2 (GSS) Given a manifold $\Sigma$, that is decomposed as $\Sigma=\Sigma_{+} \cup \Sigma_{-}$, where $\Sigma_{ \pm}$are bounded manifolds, such that $\partial \Sigma_{ \pm}=\Sigma_{+} \cap \Sigma_{-}=: S$; given a universal field supported on $\varphi$ and the regional fields, $\varphi^{ \pm}$supported on $\Sigma_{ \pm}$, GSS holds just in case the joint gauge-invariant contents of $\varphi^{ \pm}$is compatible with a unique gaugeinvariant content of $\varphi$. That is, the doublet of regional physical (i.e. gauge-invariant) states $\left(\left[\varphi_{+}\right],\left[\varphi_{-}\right]\right)$uniquely determines a valid physical state $[\varphi]$ for the field over the entire manifold $\Sigma$.

More formally, we label each legitimate/physically possible composition of the two given regional states to form a physically possible universal state by $i$, with $i$ belonging to some index set $I$, which can depend on the component states.

Thus, I will take failure of GSS to corresponds to the set $I$ having more than one element. ${ }^{18}$ We will call this Global Non-Supervenience on Regions, but keep the

\footnotetext{
${ }^{17}$ For the relation to Myrvold's "patchy separability" see footnotes 5 and 38.

${ }^{18}$ Formally, we could have $I=\emptyset$, if the two states are incompatible. As in the more general case of subsystems (as opposed to regions), I will not countenance the possibility that the relation between universe and regional physical states is one-to-many-i.e. that a single universe leaves the state on its constituent region undetermined - nor that there are valid physical states of the universe whose restrictions to regions are not themselves physically valid (I can see no plausible scenario in which that occurs).
} 
label of the more general nomenclature for subsystems ( $\neg$ GSS). Thus, denoting the gauge-equivalence class by square brackets, indicating the $i$ th composition of states by $\cup_{(i)}$ and the resulting universal physical state as $\left[\varphi_{(i)}\right]$, ${ }^{19}$ we write

$$
\left[\varphi_{(i)}\right]=\left[\varphi^{+}\right] \cup_{(i)}^{S}\left[\varphi^{-}\right], \quad i \in I \quad \text { with } \quad\left[\varphi_{(i)}\right] \neq\left[\varphi_{\left(i^{\prime}\right)}\right] \quad \text { iff } \quad i \neq i^{\prime}
$$

or, in terms of the standard Yang-Mills potential and matter fields:

$$
\left[(A, \psi)^{(i)}\right]=\left[\left(A^{+}, \psi^{+}\right)\right] \cup_{(i)}^{S}\left[\left(A^{-}, \psi^{-}\right)\right], \quad i \in I,
$$

So here $i$ is neither a spacetime index nor necessarily related to a Lie-algebra index: it is just an element of an index set $I\left(\left[\varphi^{ \pm}\right]\right)$-whose dependence on the given pair $\left[\varphi^{ \pm}\right]$will be henceforth omitted-and $\cup_{(i)}^{S}$ represents the $i$-th valid gluing, i.e. composition, of the two gauge-invariant data $\left[\varphi^{ \pm}\right]$along $S$. The global fields $\varphi$ are in the same class of differentiability as the regional ones (albeit the latter will lie on manifolds with boundary).

If $I$ is empty there is no possible gluing, i.e. the regional gauge-invariant states are incompatible and cannot conjoin into a universal physical state (regional incompatibility). If $I$ has a single element, the gluing is unique, and then there is GSS. If otherwise, i.e. if $I$ has more than one element, the universal physical state is undetermined just by the regional physical states: more information about relations between the subsystems is needed, and there is $\neg$ GSS. In this case, we will also say the universal state possesses residual variety.

Note that as it stands Definition 2 is in line with both the Galileo's ship analogy and with the idea of gauge transformations as mere re-description. Schematically: if the subsystems are 'sea' and 'ship', and there are equivalence relations, $\sim$, applicable to states of subsystems and of the whole, and given the physical (i.e. "gauge-invariant") states $\left[s_{\text {sea }}\right],\left[s_{\text {ship }}\right]$ and $\left[s_{\text {sea and ship }}\right]$, there is a many-to-one relation, encoded by the set $I$ :

$$
\left[s_{\text {sea and ship }}^{(i)}\right]=\left[s_{\text {sea }}\right] \cup_{(i)}\left[s_{\text {ship }}\right], \quad i \in I=\text { Boosts } \ltimes \text { Euclidean, }
$$

and $\left[s_{\text {sea and ship }}^{(i)}\right]=\left[s_{\text {sea and ship }}^{\left(i^{\prime}\right)}\right]$ if and only if $i=i^{\prime}$. In this case, the set $I$ that parameterizes the many-to-one relation is the (inhomogeneous) Galilean group (which is a semidirect product $(\ltimes)$ of boosts and the group of translations and rotations). The analogy states that, in general, the physical states $\left[\varphi_{\text {sea }}\right]$ and $\left[\varphi_{\text {ship }}\right]$ can be glued in a variety of ways.

Definition 2 is essentially relational: any variety will be solely a variety of relations between the subsystems. In the ship case, this variety is classified by Galilean transformations, i.e. $I$ has a 1-1 correspondence with the Galilean group.

In the following, we will see that there are circumstances in which Yang-Mills subsystems indeed admit transformations with DES due to $\neg$ GSS in this full sense. Namely, for certain regional gauge-invariant data which can be glued together i.e. composed to give a physically possible universal state, there may remain a residual

\footnotetext{
${ }^{19}$ Even though each $i$ will represent a different physical state, we avoid putting the subscript outside of the equivalence class, because there is only one equivalence class of $\varphi$; it can have no indexing.
} 
variety of universal gauge-invariant data obtained from this gluing. This variety is parametrized by rigid transformations, not malleable transformations, and is encoded by the external action of a finite-dimensional Lie group on a subsystem. In some circumstances this group will act regionally in each subsystem, but in others, the group and its action only have a natural interpretation intrinsic to the boundary between the regions. In the latter case, that can only occur in the presence of malleable symmetries, I will argue that $\neg$ GSS does not have a natural interpretation in terms of DES.

To sum up, in some cases there is a 'regional rigid symmetries'-worth of universal states which are regionally gauge-equivalent to each other but have a relational physical distinctness, i.e. are physically distinct (not universally gauge-equivalent) due to relational differences. The particular structure of $I$ will be that of a rigid subgroup of the set of gauge transformations, but it will depend on the regional field content in each case. It is the field content which identifies the rigid subgroups of the full infinite-dimensional malleable group that have DES.

In Yang-Mills theory, $\neg$ GSS is associated with DES only in conjunction with those conditions which are necessary for the existence of conserved global charges, as related to the rigid subgroup $I .{ }^{20}$ This procedure thus establishes a link between what is known as an indirect consequence of gauge - the conservation of charges - and a 'direct' one (DES).

\section{When global supervenience fails: finding $\neg$ GSS}

In this section I will explore the definition of DES as under-determination of universal gauge-invariant data from regional gauge-invariant data. That is, I will explore DES according to a failure of global supervenience on subsystems ( $\neg$ GSS) as described in Definition 2. I will illustrate this for Abelian gauge theories (i.e. electromagnetism with a scalar field). ${ }^{21}$ For this simple case, I will explicitly show that the whole state is underdetermined by the regional states, and that the ensuing variety of universal

\footnotetext{
${ }^{20}$ Namely, the association will obtain only for reducible configurations-those which have stabilizing gauge transformation (analogous to non-trivial Killing fields for a spacetime metric)—in which case $I$ is the group of reducibility parameters (analogous to the isometry group of a spacetime metric) (Barnich \& Brandt, 2002).

${ }^{21}$ I will only discuss the non-Abelian case in the Appendix. In that case, two concessions must be made: 1) due to the non-linear character of the theory, gluing takes place at the perturbative level, and so we must specify which underlying configuration is to suffer the perturbations. (So the index set $I$ would have to be written as $I\left(\left[\varphi^{ \pm}\right]\right)$as envisaged just after Eq. 2.3). Nonetheless, the formalism transforms covariantly with respect to gauge transformations of the perturbed configuration and one is able to retain, for the non-Abelian context, all the interesting results obtained in this Section. This sounds like the BRST treatment of gauge theories (see Henneaux and Teitelboim (1992) for a review), by which one retains global transformations even if one eliminates the degeneracy in the propagator of the theory through a perturbative gauge-fixing. And indeed, the tools used in this work in the non-Abelian context recover the properties of BRST ghosts; see Gomes and Riello (2017) and o(Gomes et al., 2019, Sec 3.1) for more on this recovery. 2) Only in the non-Abelian case is there a possibility to have boundary stabilizers of the gauge potential that do not extend into the bulk of the regions. Thus I will leave to Appendix A.3.2 further discussion about why the physical variety associated to these stabilizers should not be taken to have DES.
} 
states is equivalent to a copy of (i.e. is parametrized by) the charge group (U(1)). I will thus prove my main claim that there is a regional (or subsystem) rigid group of symmetries with (relational) DES, emerging from residual variety, as per Definition 2 .

I will organize this section as follows. Section 3.1 will introduce the necessary notation. Section 3.2 sets up the remaining tools for the procedure, giving an introduction to our use of gauge-fixing and gluing. In Section 3.3 I describe precisely how 'external sophistication' is deployed to facilitate the gluing of the regional physical states. In that section, I vindicate my main claims on the connection between supervenience and relational DES: viz, that there is a rigid group of regional symmetries parametrizing by the residual variety of universal physical states which are composed by identical regional physical states.

\subsection{General notation}

We are given a manifold $\Sigma$, which for our illustrative purposes in this Subsection will represent a space(time) endowed with an Euclidean metric. ${ }^{22}$ I will also assume $\Sigma$ is closed, that is, compact and without boundary. Given a charge group $G$, i.e. the finitedimensional Lie-group characterizing the theory, the group of gauge transformations is $\mathcal{G}=C^{\infty}(\Sigma, G)$. The gauge field $A$ and its gauge-transformed $A^{g}$ are given by Lie-algebra valued space(time) 1 -forms.

In the main text of the paper, I will only consider the simpler case of Maxwell electrodynamics coupled to scalar Klein-Gordon theory. Thus, for the Abelian case, the structure, or charge, group is $G=U(1)$ and we write

$$
A^{g}=A+i \operatorname{grad}(g) \text { and } \psi^{g}=\exp (i g) \psi .
$$

Here $A \in C^{\infty}\left(T^{*} \Sigma, \mathbb{R}\right)$ and $g \in C^{\infty}(\Sigma, \mathbb{R})=C^{\infty}(\Sigma, \operatorname{Lie}(G))=: \operatorname{Lie}(\mathcal{G})$, an infinitesimal gauge transformation. More generally, $A$ would be a smooth Liealgebra valued 1 -form on $\Sigma$, but here the Lie-algebra is $\mathfrak{u}(1) \simeq \mathbb{R}$. For our context and purposes-finding $\neg$ GSS in the Abelian case-the distinction between the Liealgebra and the Lie-group is unimportant, and so I will work with the Lie-algebra $u(1)$ as opposed to the group $U(1) .^{23}$ The non-Abelian case is treated in the Appendix.

I will also assume that the manifold $\Sigma$ is endowed with a Riemannian metric, $g_{i j}$, and that it is decomposed into two regions (cf. Fig. 1): $\Sigma_{ \pm}$, with boundary $\partial \Sigma_{ \pm}=$: $S=\Sigma_{+} \cap \Sigma_{-}$.

The $\Sigma_{-}$piece is what is usually labeled 'the environment', but here will play the role of another subsystem (as for relational DES; cf. footnote 10). For now, each of

\footnotetext{
${ }^{22}$ The Euclidean metric provides a simpler interpretation of the results on gluing, in the next section. See Gomes and Riello (2019) for more on this topic, and for how one can import the relevant results to the Lorentzian signature, "3+1" context. In any case, the philosophy of physics literature on DES ignores the contrast between Euclidean and Lorentzian signatures.

${ }^{23}$ In the Abelian case we will be mostly concerned with, the relation between the Lie algebra element $g \in \mathfrak{u}(1)$ and the group element, $\xi \in G$, is: $g=-i \ln \xi$, or $\xi=\exp (i g)$. This translation can be applied at any point in the following computations. Using the Lie-algebra rather than group is useful in translating our results to the non-Abelian case, since there we cannot work directly with the group.
} 


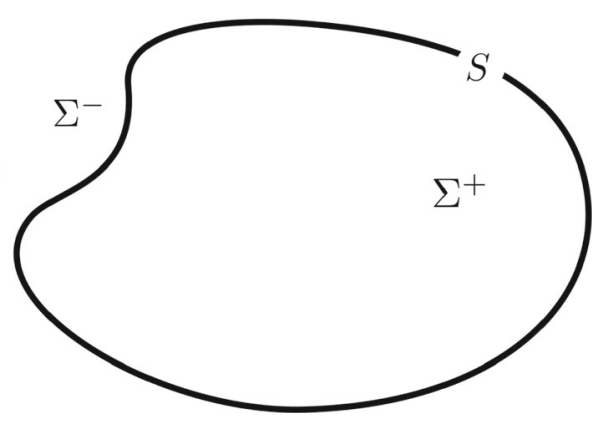

Fig. 1 The two spacetime regions $\Sigma^{ \pm}$, separated by a manifold with codimension one, $S$. Here $\Sigma$ is $\mathbb{R}^{2}$, the plane on which the figure is drawn

$\Sigma, \Sigma_{ \pm}$is assumed topologically trivial; and, if any of these manifolds is not compact, then all the fields on them will be restricted to have some suitably fast fall-off rate. ${ }^{24}$

I will denote the regional, unquotiented configuration spaces of each field sector (gauge field $A$, matter field $\psi$, and doublet (gauge and matter fields) $\varphi$ ) as $\mathcal{A}_{ \pm}$, $\Psi_{ \pm}$and $\Phi_{ \pm}$(respectively). I will omit the subscript \pm for the corresponding universal configuration spaces (i.e. $\mathcal{A}, \Psi, \Phi$, respectively). The restricted groups of gauge transformations (i.e. smooth maps from regions of the manifold into $G$ ) will be denoted in analogous fashion: $\mathcal{G}_{ \pm}=C^{\infty}\left(\Sigma_{ \pm}, G\right)$, and all abstract quotient spaces are denoted by square brackets, as in $\left[\Phi^{ \pm}\right]:=\Phi^{ \pm} / \mathcal{G}_{ \pm}$, and $[\Phi]:=\Phi / \mathcal{G}$.

If the fields compose smoothly, the left hand sides in the following equation are both smooth fields:

$$
A=A_{+} \Theta_{+}+A_{-} \Theta_{-}, \quad \text { and } \quad \psi=\psi_{+} \Theta_{+}+\psi_{-} \Theta_{-} .
$$

Here $\Theta_{+}$and $\Theta_{-}$are the characteristic functions of the regions $\Sigma_{+}$and $\Sigma_{-}$i.e. they are distributions: unity in the region, and zero outside, with some conventional value at the boundary which is immaterial for our purposes. Smoothness requires equality of the following quantities at $S:\left(\partial^{n} A_{+}\right)_{\mid S}=\left(\partial^{n} A_{-}\right)_{\mid S}$ and mutatis mutandis for $\psi$ in place of $A$; where the superscript $n$ denotes all derivatives: first order, second order, i.e. $n=1,2$, etc.; and for any combination of independent directions, i.e. $x, y, z$ parametrizing $\Sigma$. We will understand all equalities at $S$, written for simplicity like $A_{+\mid S}=A_{-\mid S}$, to be such an equality.

\footnotetext{
${ }^{24}$ In Figure 1, one could think of $\Sigma_{-}$as a collar around $\Sigma_{+}$; this would not block our treatment. The only complication would be to then consider further boundary conditions on $\Sigma_{-}$, and so on. We therefore restrict our attention to the case where $\Sigma_{-}$encompasses the "rest of the universe", i.e. the entire "environment" in the language of Greaves and Wallace (2014).
} 


\subsection{DES as $\neg$ GSS: set-up}

At first sight, the question we face in this Section is much simpler than the previous literature's construals of DES: how do we make sense of the equation (cf. Eq. 2.2):

$$
\left[\varphi_{(i)}\right]=\left[\varphi^{+}\right] \cup_{(i)}^{S}\left[\varphi^{-}\right], \quad i \in I \quad ?
$$

But there are two related obstacles.

First, the elements of $[\mathcal{A}]$, being orbits of the gauge field space $\mathcal{A}$ under the action of $\mathcal{G}$, are not directly parametrizable. Second, the only criterion for gluing quotients employs representatives, i.e. elements of $\mathcal{A}$. That is, there is no composition of physical states that is not formulated in terms of the composition of representatives.

The first step in overcoming these issues is to "gauge-fix": that is, to select a class of elements of $\mathcal{A}$ which uniquely represent elements of $[\mathcal{A}]$ (cf. (Gomes, $2019 \mathrm{Sec}$. $2)$ ). A selective class fixes further conditions which the representatives must satisfy. The second step will consist in exploiting external sophistication, as advocated in Section 1.2.

Given $\left[A_{ \pm}\right] \in\left[\mathcal{A}_{ \pm}\right]$, for any two representatives $A_{ \pm} \in \mathcal{A}$ of $\left[A_{ \pm}\right]$, the condition of composition - the conditition that must be satisfied by a pair of fields $A_{ \pm}$in order that they can be composed into a single field on the whole region-can then be translated into the following gluing condition: there exist gauge transformations, $g_{ \pm} \in \mathcal{G}_{ \pm}$, such that the gauge-transformed representatives smoothly join (cf. Eq. 3.1):

$$
A_{+}^{g_{+}}{ }_{\mid S}=A_{-}^{g_{-}} \Rightarrow\left(A_{+}-A_{-}\right)_{\mid S}=\operatorname{grad}\left(g_{+}-g_{-}\right)_{\mid S} .
$$

If there are also matter fields that are non-zero on $S$, the corresponding relation to Eq. 3.4 is:

$$
\psi_{+}^{g_{+}}\left|S=\psi_{-}^{g_{-}}\right| S \Rightarrow \exp \left(i g_{+}\right) \psi_{+\mid S}=\exp \left(i g_{-}\right) \psi_{-} \mid S .
$$

To ensure that we retain the full physical content of the regional states and the capacity to relate arbitrary configurations, there must be no prior restrictions on the gauge transformations at the boundary. ${ }^{25}$ This is crucial, both conceptually-why should the "redundant descriptive fluff" at the boundary be any different than in the bulk? - as well as technically. Our focus on gauge-invariant quantities thus allows a pure gauge discontinuity at the boundary.

In other words, from the perspective of the subsystem-instrinsic informationas in condition 2 of Definition 1 - the only criteria for the composition of regional representatives is whether under some appropriate regional gauge transformations the resulting doublets of regional representatives smoothly compose.

\footnotetext{
${ }^{25}$ While such a truncation is standard in the literature concerning gauge theory in asymptotic regions (cf. Regge and Teitelboim (1974), Balachandran et al. (1996), Giulini (1995), and Strominger (2018)), in that context there are no subsystems that should be glued back together, and the environment is not on a par with the subsystem. See Gomes (2020) for a treatment of finite regions with such an assumption, there called 'the externalist notion of boundary' and Riello (2019) for a recovery of the asymptotic results, including the so-called soft charges, using the present framework.
} 


\subsection{Reduction, sophistication, and gluing}

This is the most important technical Section of the paper, in which I illustrate the conceptual tools developed so far. In Section 3.3.1, I will show how one reduces, or fixes a selective class of representatives for the gauge fields, through a gaugefixing. ${ }^{26}$ In Section 3.3.2 I will describe how external sophistication is important for gluing. And finally, in Section 3.3.3 I describe the residual physical variety.

\subsubsection{The projection $h$ introduced}

Given the regions $\Sigma_{ \pm}$, we will consider two states, $\varphi^{ \pm}=\left(A^{ \pm}, \psi^{ \pm}\right)$. We need to uniquely characterize the physical content of such states.

Let us first focus on the gauge-fields, $A^{ \pm}$. We will return to the matter fields in Section 3.3.3. Thus we must fix unique representatives of $A^{ \pm}$, through a projection: ${ }^{27}$

$$
\begin{aligned}
h^{ \pm}: \mathcal{A}_{ \pm} & \rightarrow \mathcal{A}_{ \pm} \\
& A_{ \pm} \mapsto h^{ \pm}\left[A_{ \pm}\right]=: h_{A}^{ \pm}
\end{aligned}
$$

where $h_{A}^{ \pm}$will uniquely represent (i.e. is in the image of all elements of) the equivalence class $\left[A_{ \pm}\right]$.

But we must maintain our ability to describe the possible gluing of the $h_{A}^{ \pm}$through an analogue of Eq. 3.4. Thus $h_{A}^{ \pm}$can be gauge-invariant intrinsically—allowing internal reduction - while still allowing gauge transformations to act, "extrinsically", on them-enforcing external sophistication. That is, in the following, we can endorse 'reduction' (cf. Section 1.2) for determining the regional and universal physical content. But, to describe gluing, it is mandatory to endorse 'external sophistication': i.e. allowing all the different representations of the same regional physical content to be counted as isomorphic but not identified from the outside view.

Technically, these demands imply we should look for a projection $h_{ \pm}: \mathcal{A}_{ \pm} \rightarrow$ $\mathcal{A}_{ \pm}$, as opposed to a reduction, red $_{ \pm}: \mathcal{A}_{ \pm} \rightarrow\left[\mathcal{A}_{ \pm}\right]$. In Gomes (2019) and Gomes (2020) the construal of a gauge-fixing as a projection $h$, and not as a quotient, was argued to be fundamental for the gluing of regions: for both $h_{ \pm}$and red $_{ \pm}$are required to be gauge-invariant with respect to gauge-transformations on the common domain, $\mathcal{A}_{ \pm}$, i.e. $\operatorname{red}_{ \pm}\left(A^{g}\right)=\operatorname{red}_{ \pm}(A)$ as well as $h_{ \pm}\left(A^{g}\right)=h_{ \pm}(A)$, but only the projection $h_{ \pm}$allows further transformations to be enacted on its range.

In order to establish a direct correspondence between regional and universal physical states, we need to find unique representatives first at the regional level, glue, and

\footnotetext{
${ }^{26}$ In the Abelian case, the covariance property under transformations of the perturbed configuration need not be flagged explicitly, cf. footnote 27 below.

${ }^{27}$ Here $h$ stands for horizontal. Although we will not need to introduce the entire field-space principal fiber bundle formalism here (Gomes \& Riello, 2019), this is where 'horizontal' comes from: horizontal directions are essentially a choice of non-gauge directions in field space transforming covariantly along the fiber. The word 'horizontal' is more appropriate in the non-Abelian case: in the Abelian, horizontal directions are integrable, and correspond rather to a (covariant) foliation of the field-space $\mathcal{A}$, i.e. to a $\mathcal{G}$-covariant family of gauge-fixings.
} 
then find unique representatives of the universal state. That is, even once we have established unique representatives of the regional physical state, there are many ways of gluing (as we will see in Section 3.3.2 below), and this plethora will give rise to many possible universal representatives of the state. But $\neg$ GSS refers to the universal physical state as well as to the regional physical states. Thus, after gluing, we need to resolve the mapping between regional and universal physical states, and so we need to determine which universal physical states are formed from the gluing of which regional physical states. That is, we need to eliminate the plethora of possible extrinsic gauge transformations - that provide a redundant representation of the universal physical state-as well as the intrinsic ones, that provide a redundant representation of the regional physical states.

To discern the possible universal physical states, we apply the corresponding gauge fixing for closed-compact without boundary-regions. In the absence of boundaries, i.e. for the universal representative state $A$, instead of Eq. 3.6 we have:

$$
\begin{gathered}
h: \mathcal{A} \quad \rightarrow \mathcal{A} \\
A \mapsto h[A]=: h_{A} .
\end{gathered}
$$

Let us add some detail to this procedure.

\subsubsection{Internal reduction and external sophistication: option (c) realized.}

First, without loss of generality, we can find linear projections $h^{ \pm}: \mathcal{A}_{ \pm} \rightarrow \mathcal{A}_{ \pm}$such that their images satisfy:

$$
\left\{\begin{array}{l}
\operatorname{div}\left(h_{A}^{ \pm}\right)=0 \\
s \cdot h_{A}^{ \pm}=0
\end{array}\right.
$$

where $s$ is the normal to the boundary $S$, and $\cdot$ is induced by the inner product on $\Sigma$. It might seem surprising that we can restrict our attention to such constrained boundary conditions on $A_{ \pm}$and yet still encompass the entire gamut of possible regional physical states. The reason for this is that any other state-including ones with boundary behavior different from Eq. 3.6- differs from such a $h_{A}^{ \pm}$by a unique regional gauge transformation; again, a regional gauge transformation that is possibly non-trivial at the boundary. Thus we define the unique regional physical representatives through the projection corresponding to Eq. 3.8:

$$
\left[A_{ \pm}\right] \equiv h_{A}^{ \pm}:=A_{ \pm}+i \operatorname{grad}\left(\sigma_{ \pm}\left[A_{ \pm}\right]\right)
$$

where we denoted the relation of being $1-1$, or being uniquely represented by, or being equivalent to, by $\equiv$.

Here the $\sigma_{ \pm}$act as subsystem-intrinsic gauge transformations. More specifically, given $h^{ \pm}: \mathcal{A}_{ \pm} \rightarrow \mathcal{A}_{ \pm}$, where domain and range are seen as distinct, but isomorphic, spaces, a subsystem-intrinsic gauge-transformation is just a gauge-transformation acting on the domain of $h$. It maps between members of the same equivalence class. Here $\sigma_{ \pm}\left[A_{ \pm}\right]$is a subsystem-intrinsic gauge transformation that takes any $A_{ \pm}$to its unique representative satisfying Eq. 3.8: $\sigma_{ \pm}$enacts the projection operation. 
The label 'intrinsic' stands in opposition to 'extrinsic'. Intrinsic gauge transformations are all that is needed for a unique description of the entire Universe, since there is nothing external to the entire Universe.

Therefore we can now distinguish two sorts of action of $\mathcal{G}$ : a subsystem-intrinsic action and a subsystem-extrinsic one. Subsystem-intrinsic transformations will map between the members of the same equivalence class, whereas the extrinsic ones act as transformations between the representatives of these equivalence classes. ${ }^{28}$

But if we have more than one subsystem and we want to satisfy the gluing condition Eq. 3.4, we may need to change the representative of the equivalence class $\left[A_{ \pm}\right]$-from the outside, as it were.

For that, we need to define subsystem-extrinsic gauge transformations $g^{\text {ext }}$, as those transformations which act on the range of $h$ as

$$
h_{A}^{ \pm} \mapsto h_{A}^{ \pm}+i \operatorname{grad}\left(g_{ \pm}^{\text {ext }}\right) .
$$

Of course such a transformed field need no longer satisfy Eq. 3.8.

That is, subsystem-intrinsic gauge transformations are defined as those acting on the field configurations in the domain of the projection, whereas the subsystem extrinsic act on its range. Once we have eliminated redundancy and fixed a 1-1 correspondence with $\left[A_{ \pm}\right]$, the image of $h^{ \pm}$, i.e. $h^{ \pm}\left[\mathcal{A}_{ \pm}\right] \subset \mathcal{A}_{ \pm}$, is invariant with respect to gauge transformations acting on its domain, but we can still change representatives by acting on its range, $\mathcal{A}_{ \pm}$.

Gluing We are given the physical content of the regional configurations as (in terms of) their projected representatives $h_{A}^{ \pm}$, and while these representatives $h_{A}^{ \pm}$might not smoothly join, they may still jointly correspond to a physically possible universal state. The existence of subsystem-extrinsic gauge transformations smoothening out the transition between $h_{A}^{+}$and $h_{A}^{-}$is a necessary and sufficient condition for their compatibility.

That is, the $h_{A}^{ \pm}$determine whether they can be smoothly joined by subsystemextrinsic gauge transformations. Following Eq. 3.4, the condition is that subsystemextrinsic gauge transformations $g_{ \pm}^{\text {ext }}$ exist such that (in spacetime index-free nota$\left.\operatorname{tion}^{29}\right)$ :

$$
\left(h_{A}^{+}-h_{A}^{-}\right)_{\mid S}=i \operatorname{grad}\left(g_{+}^{\text {ext }}-g_{-}^{\text {ext }}\right)_{\mid S} ;
$$

which is the appropriate rewriting of the gluing condition Eq. 3.4.

However, as mentioned, this is not enough to establish a direct correspondece between universal and (the doublet of) regional physical states: although we have the

\footnotetext{
${ }^{28}$ It is instructive to compare the two possibilities of action of $\mathcal{G}$ to the use of homotopy type theory (HoTT) in gauge theory, as advocated by Ladyman (2015). Ladyman says HoTT "both (a) distinguishes states conceived of differently even if they are subsequently identified, and (b) distinguishes the identity map from non-trivial transformations that nonetheless might be regarded as delivering an identical state". Here we have two sorts of transformations: the subsystem-intrinsic one, $A \mapsto A^{g}$, which does not change $h[A]$ — satisfying Ladyman's (b)—, and the subsystem-extrinsic one, that does the work of Ladyman's (a). ${ }^{29}$ Using indices, the equation is: $\left(h_{+}^{\mu}-h_{-}^{\mu}\right)_{\mid S}=i \partial^{\mu}\left(g_{+}^{\text {ext }}-g_{-}^{\text {ext }}\right)_{\mid S}$.
} 
physical representatives on the regional side, we do not yet have them on the universal side of the correspondence.

\subsubsection{Establishing the main claims}

Finally, we must know which of these gluings give rise to physically distinct universal configurations. Thus, we need to successively eliminate the redundancy of subsystem-intrinsic and extrinsic gauge transformations and get a unique representative of the regional and glued universal state.

To add details to the previous considerations: at the universal level, Eq. 3.8 reduces to the familiar statement that the condition

$$
\operatorname{div}\left(h_{A}\right)=0 .
$$

This is a bona-fide (partial) gauge condition, called the (Euclidean) Lorentz gauge, or, in the non-Abelian setting, the (perturbative) covariant Landau gauge. It is 'partial' because a different choice, related to $h$ by a constant shift, would still satisfy Eq. 3.12. It implies that the representative of the universal physical state is only determined up to a global phase shift. This will be important in what is to come. It is also important to notice that the particular choice of gauge is immaterial for the forthcoming theorems (cf. (Gomes \& Riello, 2019, Sec.6.1.2)).

We know that each $h$ and $h^{ \pm}$yields a unique element in each orbit because of the way the projection $h: \mathcal{A} \rightarrow \mathcal{A}$ works by exploiting gauge transformations. For instance, in the universal case, for $\mathrm{U}(1)$ :

$$
h[A]:=A+i \operatorname{grad}\left(i \nabla^{-2}(\operatorname{div}(A))\right)=A^{\sigma[A]},
$$

where $\nabla^{-2}$ is the inverse operator to the Laplacian (a Green's function), ${ }^{30}$ and the functional

$$
\sigma[A]:=i \nabla^{-2}(\operatorname{div}(A))
$$

is the unique solution of the equation:

$$
\operatorname{div}(h[A])=\operatorname{div}\left(A^{\sigma[A]}\right)=0 .
$$

Moreover, it is easy to see from Eq. 3.13 that the $h[A]$ satisfies $h\left[A^{g}\right]=h[A]$, $\forall g \in \mathcal{G}$. Thus $h$ is a complete, gauge-invariant functional, uniquely representing each equivalence class; we have one, and only one $h^{A}:=h[A]$ per orbit of the gauge group. And a similar $\sigma^{ \pm}$, with analogous properties, exists for for $h^{ \pm}$obeying the regional (3.8).

Putting it all together: we start being given the regional physical states, by Eq. 3.9, which we just denote by $h_{A}^{ \pm}$. These are the original $A_{ \pm}$adjusted by the subsystemintrinsic gauge transformations $\sigma^{ \pm}$enacting the projections onto the constraint surface. Now we allow extrinsic gauge transformations to act on the range of $h^{ \pm}$, as in Eq. 3.10, i.e. $h_{A}^{ \pm} \mapsto h_{A}^{ \pm}+i \operatorname{grad}\left(g_{ \pm}^{\text {ext }}\right)$. We then form a glued state:

$$
h_{A}:=\left(h_{A}^{++} i \operatorname{grad}\left(g_{+}^{\text {ext }}\right)\right) \Theta_{+}+\left(h_{A}^{-}+i \operatorname{grad}\left(g_{-}^{\text {ext }}\right)\right) \Theta_{-}
$$

\footnotetext{
${ }^{30}$ This is usually written, in the theory of differential operators on distributions, through a bi-local operator $\nabla^{-2}:=G(x, y)$, i.e. a Green's function, so that $\int G(x, y) \nabla^{2} F(x)=F(y)$.
} 
by requiring that $g_{ \pm}^{\text {ext }}$ obey Eq. 3.11, and $h_{A}$ obeys Eq. 3.12, and thus uniquely represents the physical state, i.e. demanding that $\operatorname{div}\left(h_{A}\right)=0$ (we assume the universe has no boundary).

Imposition of these conditions on Eq. 3.16 almost uniquely fixes $g_{ \pm}^{\text {ext }}\left[\left(h_{A}^{+}-\right.\right.$ $\left.\left.h_{A}^{-}\right)_{\mid S}\right]{ }^{31}$ In the U(1) case, there is an under-determination by a constant extrinsic gauge transformation in each region; let us call this indeterminacy $\left(c^{+}, c^{-}\right)$. Thus $g_{ \pm}^{\text {ext }}\left[h_{A}^{+}, h_{A}^{-}\right]$is fixed up to the addition of constants, $c_{ \pm} \in \mathbb{R}$. We note that as expressed, this is an indeterminacy at the level of the Lie-algebra; for the Lie-group, we would get $\exp \left(i c_{ \pm}\right) \in[0,2 \pi]$.

The hard work is now behind us. But to finally get to the main claims of the paper, we must still include charged matter fields in our description.

Charged matter fields First, note that the discussion so far focused on the gauge fields, A. But including matter fields is not difficult, since matter fields 'co-rotate' with $A_{ \pm}$under gauge-transformation. That is, since both the gauge and matter fields are subject to the same gauge transformations (you cannot consistently apply one gauge-transformation for $A$ and another for the matter field $\psi$ ), the gauge representative of matter fields just get "taken for the ride" by the fixing of $\sigma[A]$. That is, when the gauge transformations projecting onto the gauge-fixing surface, $\sigma_{ \pm}$, are unique, a given doublet, $\varphi_{ \pm}=\left(A_{ \pm}, \psi_{ \pm}\right)$is also projected to a unique representative:

$$
\left(h_{A}^{ \pm}, h_{\psi}^{ \pm}\right):=\left(A_{ \pm}+i \operatorname{grad}\left(\sigma_{ \pm}\left[A_{ \pm}\right]\right), \exp \left(i \sigma_{ \pm}\left[A_{ \pm}\right] \psi_{ \pm}\right)\right.
$$

In the asymptotic flat case, the universal functional $\exp (i \sigma[A]) \psi$ is known as the "Dirac dressed electron" (Dirac, 1955). It is an electron that is "dressed" by an appropriate Coulombic tail, rendering the electron also gauge-invariant (as can be easily checked from the gauge-covariant transformation properties of $\sigma$ ). The function $h_{ \pm}$ is known as the "radiative" projection of the photon. Here we have extended both notions to finite bounded regions (see Gomes et al. (2019) and Gomes and Riello (2019)).

Since we find unique representatives for the full regional physical field content, we can identify (using the notation' $\equiv$ ') the physical content with its unique representative:

$$
\left[\varphi_{ \pm}\right] \equiv\left(h_{A}^{ \pm}, h_{\psi}^{ \pm}\right)
$$

and take these as the starting point for gluing.

\footnotetext{
${ }^{31}$ In both Abelian and non-Abelian case, $g_{ \pm}^{\text {ext }}$ depends on $h_{A}^{ \pm}$only through their difference at the boundary: $\left(h_{A}^{+}-h_{A}^{-}\right)_{S}$ (in the non-Abelian case, each $g^{\text {ext }}$ depends also on its respective regional gauge field, e.g. $\left.g_{+}^{\text {ext }}\left[A_{+},\left(h_{A}^{+}-h_{A}^{-}\right)_{\mid S}\right]\right)$. For illustration purposes, I display the solution here:

$$
\ln g_{ \pm}^{\text {ext }}=\zeta_{( \pm)}^{ \pm \Pi} \quad \text { with } \quad \Pi=\left(\mathcal{R}_{+}^{-1}+\mathcal{R}_{-}^{-1}\right)^{-1}\left(\left(\nabla_{S}^{2}\right)^{-1} \operatorname{div}_{S}\left(h_{A}^{+}-h_{A}^{-}\right)_{S}\right)
$$

where the subscript $S$ denotes operators and quantities intrinsic (i.e. pulled-back) to the boundary surface $S$ (and since normal components of $h_{A}^{ \pm}$match, $\left.\left(h_{A}^{+}-h_{A}^{-}\right)_{\mid S}=\left(h_{A}^{+}-h_{A}^{-}\right)_{S}\right) ; \zeta_{( \pm)}^{u}$ is a harmonic function on (respectively) $\Sigma^{ \pm}$with Neumann boundary condition $\partial_{n} \zeta_{( \pm)}^{u}=u$, and $\mathcal{R}$ is the Dirichlet-to-Neumann operator. For the meaning of these operators, and also the analogous solution for the general non-Abelian Yang-Mills gauge theories, see (Gomes \& Riello, 2019, Sec. 6).
} 
Inputting Eq. 3.18 into the universal state Eq. 3.16 and solving for Eq. 3.12, we obtain $g_{ \pm}^{\text {ext }}\left[h_{+}, h_{-}\right]+c_{ \pm}$. Thus, we have:

$$
[\varphi]_{\left(c^{+}, c^{-}\right)} \equiv\left(\left(h_{A}^{++i} \operatorname{grad}\left(g_{+}^{\mathrm{ext}}\right)\right), \exp i\left(g_{+}^{\mathrm{ext}}+c_{+}\right) h_{\psi}^{+}\right) \Theta_{+}+(+\leftrightarrow-)
$$

where the term $(+\leftrightarrow-)$ is identical to the first, mutatis mutandis for - replacing + , and I have omitted the dependence of $g_{ \pm}^{\text {ext }}$ on $h_{A}^{ \pm}$. The important point to understand is that, although the degenaracy in gluing, $c_{ \pm}$, has no effect on the gauge field, it will add a phase, or rotate, the matter part.

But now we must consider two cases: either the $\psi_{ \pm}$vanish at $S$, or they don't.

Assume first that $\psi_{ \pm}$vanish at $S$. We then have no further constraints and thereby obtain a 2-parameter family of universal states, Eq. 3.19, parametrized by $\exp \left(i c_{ \pm}\right)$. In other words, given regional physical states $\left[\varphi_{ \pm}\right]$, here a conjunction of e.g. a transverse projection of the photons and a Dirac dressing of the charges, we can glue them to form a two-parameter collection of universal states.

Naively, this would give us two copies of U(1), parametrizing the universal physical states that are compatible with the regional physical contents. But of course, if $c_{+}=c_{-}$, we have a global constant phase shift (which precisely matches the expected left-over under-determination of the universal physical state $[\varphi]$ by Eq. 3.12) which does not change the universal physical state. Therefore, we are left with a residual physical variety parametrized by the difference, $\bar{c}:=c_{+}-c_{-}$, which is insensitive to any global phase shift. To sum up, if the matter fields vanish at the boundary, we obtain a residual variety that is isomorphic to a single copy of U(1) and in fact can be identified with relative, regional, rigid phase shifts.

Assume now that $\psi_{ \pm}$do not vanish at $S$. Then not every boundary value is allowed: the two sides must differ by a phase at the boundary, and this phase difference needs to match the gauge transformation required to glue the gauge potentials. That is, by Eq. 3.5,

$$
\exp i\left(\left(g_{+}^{\mathrm{ext}}+\sigma_{+}\right)-\left(g_{-}^{\mathrm{ext}}+\sigma_{-}\right)+\left(c_{+}-c_{-}\right)\right) \psi_{+\mid S}=\psi_{-\mid S}
$$

where $\left(g_{+}^{\text {ext }}+\sigma_{+}\right)-(-\leftrightarrow+)$ is a fixed functional of the gauge fields, and $c_{ \pm}$, which were entirely under-determined previously by the gauge fields $A$, will now completely fix the quantity $\left(c_{+}-c_{-}\right)$by Eq. 3.20. Thus, if the matter fields do not vanish at the boundary (and are compatible with each other and with the gauge fields, satisfying (3.20), there is no variety left, since the compatibility (3.20) completely fixes the difference $\bar{c}$.

Finally, as a corollary of these constructions, we are able to state our main result of this Section for gluing physical states:

Theorem 1 (Rigid variety for U(1)) For electromagnetism as coupled to a KleinGordon scalar field in a simply-connected universe: given the physical content of two regions, $\left[\varphi_{ \pm}\right]$, for matter vanishing at the boundary but not in the bulk of the regions, the universal state is underdetermined, resulting in a residual variety parametrized by an element of $\mathrm{U}(1)$. In the notation of Eq. 2.2 and Definition 2:

$$
\left[\varphi_{(i)}\right]=\left[\varphi_{+}\right] \cup_{(i)}^{S}\left[\varphi_{-}\right], \quad i \in I \simeq \mathrm{U}(1)
$$


where the particular action of $\mathrm{U}(1)$ is that which leaves the gauge-fields invariant, but not the matter fields. ${ }^{32}$

Thus we have found, in the case of electromagnetism coupled to a scalar field, a rigid variety through $\neg$ GSS, as per Definition 2 in Section 2.4. Moreover, by fixing a reference subsystem $\left(\Sigma_{-}\right)$, we can construe this $\mathrm{U}(1)$ as acting as a group of (rigid), regional symmetries over $\Sigma_{+}$, that shift the phase of matter but do not affect the gauge potentials (as in the 't Hooft beam-splitter, ${ }^{33}$ ('T Hooft, 1980)). That is, we have constant phase shifts.

In direct analogy with Galileo's ship—where we also recognize $\neg$ GSS as encoded by the (external) action of a finite-dimensional group — we have fully vindicated our main claims: namely, that $\neg$ GSS is a source of empirically significant subsystem symmetries.

Agreed, the non-Abelian Yang-Mills case is more complicated: non-linearities render the corresponding $I$ of the equation corresponding to Eq. 3.21 dependent on the physical state. Nonetheless, our constructions are valid at a perturbative level, i.e. one needs to first fix a ground state and then perturb it. If the perturbed state is the 'vacuum', i.e. $\left[A^{*}\right]$ for $A^{*}=0$, then we recover the full Lie algebra of the gauge group through the analogue of Eq. 3.21. In that case too, the particular rigid subgroup is identified as the one that leaves the perturbations invariant but not the matter sources.

\section{Comparison with Greaves \& Wallace and Brading \& Brown}

Now that our work is done, I will draw several lines of comparison to the approaches of Brading and Brown (2004) and Greaves and Wallace (2014). Their positions were introduced briefly in Section 2.3 in sparse detail, but we will not need more than that. In Section 4.1 I briefly gloss (Brading \& Brown, 2004)'s argument against DES; it relies on an a priori condition on the possible doublet of regional gauge transformations: that they smoothly join. In Section 4.2, I briefly gloss (Greaves \& Wallace, 2014)'s arguments for DES. In Section 4.4, I will show, contra (Greaves \& Wallace, 2014), that the lack of DES for general malleable symmetries still allows for the context-dependent identification of some of their rigid subgroups-which do have DES.

\subsection{Brading and Brown: regional gauge transformations must match}

As the quotation at the end of Section 2.3 illustrates, (Brading \& Brown, 2004) take any two regional gauge transformations which fail to coincide at their common

\footnotetext{
${ }^{32}$ In this phrasing, the variety is more widely applicable, e.g. to non-Abelian fields. For the Abelian case, it just means we take the group of constant phase shifts.

${ }^{33}$ This is a beam-splitter with two arms (containing electron fields) - the equivalent of our regionsand the phases are individually shifted by a constant in each arm. This phase difference can alter the interference pattern, and thus, it serves as an example of a subsystem gauge transformation with DES.
} 
boundary to be disallowed. In discussing the t'Hooft beam splitter (cf. footnote 33), they conclude:

"The only remaining option is to consider a region where the wavefunction can be decomposed into two spatially separated components, and then to apply local gauge transformation to one region (i.e. to the component of wavefunction in that region, along with the electromagnetic potential in the region) and not to the other. But then either the transformation of the electromagnetic potential results in the potential being discontinuous at the boundary between the 'two subsystems', in which case the relative phase relations of the two components are undefined (it is meaningless to ask what the relative phase relations are), or the electromagnetic potential remains continuous, in which case what we have is a special case of a local gauge transformation on the entire system." (p. 656)

Brading and Brown (2004) are right in one respect: once you have a universal configuration one can not apply gauge transformations which are discontinuous (e.g. produce a delta function) at the interface. ${ }^{34}$ On the other hand, it is also true that one could have regional configurations being acted upon by regional gauge transformations which don't match at the boundary. ${ }^{35}$ In this second instance, one aims to take the regional subsystem intrinsically and have non-matching gauge transformations applied to them before gluing; it reflects our emphasis on composition as opposed to decomposition, as described in Section 1.1.

In other words, in their quotation, Brading and Brown (2004) ignore the fact that the formulation of DES, through property 2 of Definition 1, requires only information intrinsic to a subsystem-which is why we related it to supervenience of the universe on its subsystems. All hands agree that, from the viewpoint of the universe one cannot have a gauge transformation which is discontinuous at the boundary. From this viewpoint it is true that $g_{+}=g_{\mid \Sigma_{+}}$and $g_{-}=g_{\mid \Sigma_{-}}$; i.e. that the regional gauge transformations are mere restrictions of a universal gauge transformation. And their inference is flawless: such an assumption would pre-empt any search for DES.

But the assumption is flawed. For the topic of supervenience, one starts from the regional states and then composes them. From this perspective, it is the effect of the regional gauge transformations that matter.

Greaves and Wallace (2014) spot this error, and assert that what should be fundamental is only the continuity of the glued gauge and matter fields, $A, \psi$, not of phase shifts. In their words (but my notation) (p. 83):

The key to seeing why this argument fails is noting that what is given, when we are given the pre and post-transformed states of the universe, is not a function from spacetime to the gauge group, but merely the effect of whatever transformation is being performed on the particular pre-transformation (universe) state $(\psi, A)$. And if this particular $\psi$ happens to vanish on the overlap

\footnotetext{
${ }^{34}$ See p.79 and 82 Greaves and Wallace (2014) for their endorsement of continuity of universal gauge transformations.

${ }^{35}$ See p. 83 of Greaves and Wallace (2014) for their endorsement of this point.
} 
region [the boundary $S$ ], then nothing about the corresponding gauge transformations $g_{+}, g_{-}$can be 'read off' from their effects on the wavefunction in that region [assuming they are constant near $S$ ]. It is therefore possible that the universe transformation being performed might correspond to the effect of (say) some constant gauge transformation $g_{+}$in $\Sigma_{+}$, and a different constant gauge transformation $g_{-}$in $\Sigma_{-}$, so that there is no way of patching $g_{+}, g_{-}$together to obtain a single smooth function from the whole of spacetime to the gauge group. [my italics]

I fully agree with this verdict, as far as it goes. It means one considers the effect of the regional $g_{ \pm}$on the subsystems from the intrinsic point of view. One does not take $g_{ \pm}$ as the regional restrictions of a discontinuous $g$. But I believe Greaves and Wallace (2014) do not consistently apply composition throughout their procedure, as we will shortly see.

Nonetheless, Greaves and Wallace (2014) are one step closer to an analysis based solely on physical (gauge-invariant) concepts than are Brading and Brown (2004): they take the representatives of the subsystem fields to be important, not the gauge transformations themselves. I will now develop their view, and, in particular, will parse the italicized text in their quote above.

\subsection{Greaves and Wallace: non-matching gauge transformations encode DES}

To recapitulate: Greaves and Wallace (2014) claim the relational DES transformations are in 1-1 correspondence with the following quotient of two infinitedimensional groups:

$$
\mathcal{G}_{\mathrm{DES}}^{\mathrm{GW}}(\varphi) \simeq \mathcal{G}(s \mid \partial) / \mathcal{G}_{\mathrm{Id}},
$$

where $\mathcal{G}\left(s_{\mid \partial}\right)$ are the gauge transformations of the region which preserve the state $\varphi$ at the boundary of the region, and $\mathcal{G}_{\text {Id }}$ are the gauge transformations of the region which are the identity at the boundary.

Here is the gist of the argument (reflected in the above quote) leading from Definition 1 to Eq. 4.1 (and reflected in the above quote): certain gauge transformations are not the identity at the boundary and yet they may keep particular boundary states invariant. One can use such regional transformations to obtain a different universal state, but the composition of the regional gauge transformations is not itself smooth and therefore does not count as a gauge transformation relating the initial and final universal configurations. Greaves and Wallace (2014)'s main mistake is that they implement no criteria to establish whether the regional and universal states are indeed physically distinct. As I will show below, this omission allows us to find a simple counter-example, in which Eq. 4.1 is non-trivial and yet the transformations constructed above fail to yield physically distinct states, and thus do not satisfy condition 1 of Definition 1.

In more detail, let $\left(A_{ \pm}, \psi_{ \pm}\right)$be two configurations, one in each region $\Sigma_{ \pm}$, that join smoothly, and such that $\psi_{ \pm}=0$. Therefore we have the initial universal configuration $A=A_{+} \Theta_{+}+A_{-} \Theta_{-}$.

As a condition for these representatives to smoothly compose, we must have: $A_{+\mid S}=A_{-\mid S}$ (where equality here includes equality of derivatives at $S$ ). But 
$A_{+\mid S}$ has a stabilizer: at the Lie algebra level, this is $g_{+\mid S}=c_{+} \neq 0$ such that $\operatorname{grad}\left(g_{+}\right)_{\mid S}=0$ and where $c_{+}$is some constant on $S$. Therefore, the configuration

$$
\tilde{A}:=A_{+}^{g_{+}} \Theta_{+}+A_{-} \Theta_{-}
$$

is still smooth, since $g_{+}$doesn't change the value of $A_{+}$at the boundary. But

$$
g_{+} \Theta_{+}+g_{-} \Theta_{-}=g_{+} \Theta_{+},
$$

with $g_{-}=0,{ }^{36}$ is not a smooth (infinitesimal) gauge transformation (because $g_{+}$ doesn't vanish at $S$ ). (Greaves \& Wallace, 2014) would conclude from this that $\tilde{A}$ is not a gauge-transformed $A$, and that, therefore, $\tilde{A}$ and $A$ are physically distinct. Moreover, they observe, such a $g_{+}$corresponds to an element of Eq. 4.1 and essentially, they claim, the same construction would apply for any other element of this quotient group.

But without dealing with gauge-invariant quantities, Greaves and Wallace (2014) have no warrant to conclude that $\tilde{A}$ and $A$ are physically distinct, and therefore no warrant to conclude that condition 1 of Definition 1 is satisfied. In fact, it is only true that $\tilde{A}$ as it is written, doesn't appear to be a gauge transformed $A$. But we can explicitly construct a gauge transformation relating $\tilde{A}$ and $A$ as follows: Let $\tilde{g}_{+}:=$ $g_{+}-c_{+}$. Now $A_{+}^{\tilde{g}_{+}}=A_{+}^{g_{+}}$(because the constant part has a trivial action on $A_{+}$), and so:

But now

$$
\tilde{A}=A_{+}^{\tilde{g}_{+}} \Theta_{+}+A_{-} \Theta_{-} .
$$

$$
g:=\tilde{g}_{+} \Theta_{+}+g_{-} \Theta_{-}=\tilde{g}_{+} \Theta_{+}
$$

is a smooth gauge transformation (since $\tilde{g}_{+}$vanishes at $S$ ). And therefore $\tilde{A}=A^{g}$, so $\tilde{A}$ is a gauge-transformed $A$ after all! Thus $[\tilde{A}]=[A]$ : that is, the physical, gauge-invariant, states are identical and the transformation cannot have empirical significance (condition 1 of Definition 1 fails). ${ }^{37}$

(Although the above argument was formulated explicitly for electromagnetism, its extension to the more general cases is straightforward.)

\subsection{Greaves \& Wallace's and Brading \& Brown's errors}

In my view, neither Greaves and Wallace (2014) nor Brading and Brown (2004) could have obtained the right characterization of DES, for Definition 2's characterization of DES in terms of physical i.e. gauge-invariant, states was not articulated by either group. Indeed, they both explicitly endorse GSS. On this topic, Greaves and Wallace (2014) write:

\footnotetext{
36 That is, Greaves and Wallace (2014) take $g_{-} \equiv 0$ : they take the environment gauge transformations to be the identity, or to serve 'as a reference' for the gauge transformations of the subsystem, and the original $g_{+}$to be the identity. This is a bit confusing, since stipulating the value of $g$ does not usually fix a selective class: given $A$, one cannot use this condition to assess whether $A$ belongs to the selective class. Here, since we are only interested in a counter-example to substantiate our criticism of their construal of DES, we will ignore this point (which is not an issue for our formulation through $\neg$ GSS).

${ }^{37}$ If there was matter in the bulk, our counter-example would fail, because $\psi_{+}^{\tilde{g}_{+}} \neq \psi_{+}^{g_{+}}$. But the source of such DES would be just what I described here through Theorem 1: namely, as required from the Theorem, there is a stabilizer of the gauge potential but not of the matter field.
} 
GSS': "Firstly, in doing so we make the assumption that knowing the state of the subsystem and its environment suffices to specify the state of the total system. [...] For example, it is true for Yang-Mills gauge theories in the connection formalism but not in the holonomy formalism." (p. 67)

This assumption, which I have labeled as an alternative statement of GSS, should not be confused with its converse, which, all hands agree, holds for all of these systems. Namely, the converse assumption-that the state of the whole, uniquely determines the states of the subsystems-is not under dispute since the state of the whole includes all relational information and more. But as we have stressed: given just the intrinsic physical states of the subsystems, it is not necessarily the case that there is just one way of putting them together; their conjunction may lack necessary relational information.

The exception Greaves and Wallace (2014) make for the holonomy formalism is telling here: how can physical significance depend on the choice of variables? Indeed, for us it is immaterial: for electromagnetism, it is possible, using holonomies, to recover precisely the same results as in Theorem 1. For Greaves and Wallace (2014), it fails precisely because the holonomy formalism deals with gauge-invariant observables. $^{38}$

\footnotetext{
${ }^{38}$ Here I comment on the relation between $\neg$ GSS and Myrvold's 'global patchy-non-separability' (Myrvold, 2010, p.427), which they articulated for electromagnetism in holonomy variables (cf. footnote 5). In electromagnetism, given the space of loops (smooth embeddings $\gamma: S^{1} \rightarrow \Sigma$ ), one can form a basis of gauge-invariant quantities by the holonomies, $\exp \left(i \oint_{\gamma} A\right)$ (this can be accomplished more generally for non-Abelian theories using Wilson loops, Barrett (1991)). For simply connected regions $\Sigma$ like ours, by composing regional loops $\gamma_{ \pm} \in \Sigma_{ \pm}$going in opposite directions at the boundary $S$ it is true, as Myrvold argues, that we recover the gauge-invariant holonomy corresponding to a larger loop $\gamma$ not contained in either region. Therefore any universal holonomy corresponds to a single doublet of regional holonomies. According to Myrvold, separability fails only for non-simply connected manifolds, where the holonomies of $\gamma_{ \pm}$cannot recover the universal holonomy of $\gamma$.

I have two comments to make on the relation to the present work: (i) In the absence of matter and in the Abelian setting, non-trivial topology indeed is the only source of $\neg$ GSS, and we recover Myrvold's conclusions. But with matter, we can close off curves which are not loops-which only change by gauge transformations at their ends-by placing charges to cap off the curves, thereby obtaining gauge-invariant holonomies (cf. (Gomes \& Riello, 2019, Sec. 4.3.2)). These sourced holonomies correspond to the residual variety we found for electromagnetism in Theorem 1, which occurs even for simply-connected $\Sigma$ when charged matter is present in the regions $\Sigma_{ \pm}$.

(ii) Unfortunately, Myrvold's loop composition doesn't work in the same way for the non-Abelian theory: although the appropriate regional loops themselves will compose as curves in the manifold, Wilson loops-giving the gauge-invariant content of the holonomies-involve traces, and the traces ruin the composition properties: the corresponding regional gauge-invariant quantities do not compose. Our construction (see Appendix A.2) gets around that.

The conclusions, in Myrvold's nomenclature, are then: global patchy separability fails for non-Abelian theory if and only if: the manifold is non-simply connected, or charged matter is present inside the regions and furthermore this charged matter obeys (perturbative) regional conservation laws (i.e. and the perturbed gauge-field has stabilizers).
} 


\subsection{How can a rigid subgroup have DES?}

Now it is our turn to defend our construction from one of Greaves and Wallace (2014)'s arguments, who judge it impossible that a 'global' (i.e. rigid) gauge transformations, being a subset of the 'local' (i.e. malleable), may acquire DES, while none of the malleable do.

As expressed in Eq. 4.1 for relational DES, (Greaves \& Wallace, 2014) only claim a group isomorphism between $\mathcal{G}_{\mathrm{DES}}^{\mathrm{GW}}$, whatever it may be, and a quotient group, "empirical symmetries correspond 1-1 [...] to elements of a quotient group" (p. 75). ${ }^{39}$ But Greaves and Wallace (2014) do not see the quotient nature of their result as problematic. In fact, they see it as exonerating their notion of relational DES from the charges they make against the orthodox view on DES:

Nothing like the local/global distinction tells us which symmetries can in general have empirical significance and, in particular, it is false that local symmetries are in general unobservable. (p. 62)

(the italics are mine). That is, their accusation against the orthodox view is that it attributes relational DES only to certain rigid subgroups of malleable groups.

Besides my criticisms of GSS' (that is, that regional states should uniquely define the universal state) and of the lack of a gauge-invariant criterion for DES, made in Section 4.3, I have one more disagreement, with the quote from (Greaves \& Wallace, 2014, p. 62), reproduced just above. Contrary to what they affirm, it is precisely the rigid/malleable distinction that tells us which symmetries can have empirical significance. Indeed the rigid symmetries can only be meaningfully discerned from the malleable contingently, when they have physical significance, according to my proposal. Moreover, it is not false, but true, that malleable symmetries are in general unobservable. ${ }^{40}$

And these conclusions hold, even if the group of rigid symmetries, when one can be identified, is a subset of the malleable.

Admittedly: if one focuses just on the group itself, and not on its action on states, one indeed cannot "pluck out" a rigid subgroup in any meaningful way. But contra Greaves and Wallace (2014), it is entirely possible to associate DES only to certain physically meaningful rigid symmetries; the meaning is acquired through their

\footnotetext{
${ }^{39}$ The claim is based on intuitions for gauge theories in the asymptotic regime. But the situation in the asymptotic regime, or for truncated configuration spaces, is more complicated: there, one does not consider environment and subsystem to be on a par as we do for relational DES; the environment truncates the fields and the gauge transformations at the boundary of the subsystem. In that case, we can obtain a group of symmetries with DES that is isomorphic to the quotient and has no action on the subsystem states. These results, obtained in Gomes (2020, Sec 4.2.2), largely recover those presented by Greaves and Wallace (2014) (erroneously) for relational DES in Eq. 4.1, and more.

${ }^{40}$ I should clarify what 'in general' means here: for generic configurations of the non-Abelian theory, and, in the Abelian case, for configurations in which there are charges in the bulk of a region, but not in the boundary. In contrast, for Greaves and Wallace (2014), any configuration that lacks boundary charges will attribute DES to all but a meager subset of the malleable symmetries. Namely, in their mathematical treatment, the only malleable symmetries that lack DES are those that are the identity at the boundary.
} 
action on the fields. The transformations are the only ones that leave the gaugefield, but not the matter fields, invariant. ${ }^{41}$ That means that in any background, malleable transformations that don't belong to these subgroups would lack DES. That is, rigid symmetries may have DES in a given special background and yet lose that significance for a generic background. Such subgroups are "plucked out" from the surrounding malleable group by satisfying certain equations, e.g. the Killing equations, which are themselves physically significant.

It is interesting here to pick up on the quote from page 62, regarding spacetime translations. First, we must ask: what are the rigid translations and boosts in general relativity, i.e. of a generic spacetime metric? Poincaré transformations are not well-defined (i.e. geometrically defined) in a generic background metric. They are defined by Killing fields for a Minkowski metric. In that sense, that subgroup is physically distinguished, but only in particular circumstances: it is only meaningful for a Minkowski metric; ${ }^{42}$ in specific backgrounds (e.g. Minkowski), there will be a physically well-defined rigid subgroup of the malleable transformations, but this subgroup will be effaced once one moves to generic backgrounds. One need not resort to quotients to define this rigid subgroup, when the physical content of the theory will do it for you.

Such subgroups of rigid symmetries are usually called stabilizers, and the configurations they stabilize are called reducible (cf. footnotes 16 and 20). And the same concepts apply to gauge theories. In the Abelian case all $A \in \mathcal{A}$ are reducible, and they all possess the same stabilizers, namely, the constant gauge transformation. The non-Abelian case is much more similar to the spacetime case (see Appendix A.3 for a brief description): generic $A$ are not reducible, but some are. Being reducible is a physically significant fact: in the quotient space $[\mathcal{A}]$, the orbits of reducible configurations are qualitatively different than the generic orbits. ${ }^{43}$

In sum, the translations may have significance, which show up only contingently (in a Minkowski background), and which we deny to general diffeomorphisms. More importantly, to characterize this significance we can talk about translations only, without ever invoking general diffeomorphisms. That is, to talk about the physical

\footnotetext{
${ }^{41}$ Indeed, such special configurations are part of the structure of the physical quotient of the configuration space of the theory by the gauge transformations, $\Phi / \mathcal{G}$ : the quotient is not a manifold, but a stratified manifold, and the orbits whose fields are stabilized by subgroup of $\mathcal{G}$ are the 'strata'.

${ }^{42}$ If one is thinking not in terms of active diffeomorphisms, but of coordinate transformations, then indeed, one can single out translations and boosts, but only with respect to that coordinate system. More broadly, there are generically no constant gauge-transformations: they usually require a global section to be defined. One should also note that although for principal fiber bundles one has a natural action of the charge group, $G$, this is not the case for associated bundles. One cannot define a "constant" action of the gauge group: it can only be constant with respect to a given section (see Kobayashi and Nomizu (1963)).

${ }^{43}$ Note that the notion of reducibility covaries with the notion of stabilizers. Namely, if a configuration $\varphi$ is reducible, with a given stabilizer $f \in \mathcal{G}$, then for any given $g \in \mathcal{G}, \varphi^{g}$ will also be stabilized by $\mathrm{g} \mathrm{gg}^{-1}$. This qualitative difference between the orbits renders the quotient space into a stratified manifold: i.e. a space formed by a concatenation of boundaries (see Kondracki and Rogulski (1983) and Fischer (1970) and, for a philosopher-friendly description of stabilizers, and their relation to conserved charges, (Gomes, 2019, Secs. 3.3.5-7))
} 
significance of rigid symmetries one need never invoke the larger malleable group in which they can be embedded.

\section{Conclusions}

In this section we summarize in Section 5.3, but before that we gather two remaining threads: the meaning of holism vis a vis the non-locality of gauge theories, discussed in Section 5.1, and the epistemic role of subsystems, in Section 5.2.

\subsection{Whence holism?}

Gauge-invariant quantities are to some extent non-local (L9 in Earman's classificatory scheme (Earman, 1987)), which means there is in principle "room to explore" between the whole and the sum of the parts. And it turns out that the known aspects of non-locality in gauge theory, to a certain extent match the non-local aspects of $\neg \mathrm{GSS}$.

One can see this is as follows. Gauss constraints are the defining characteristic of gauge theories: they stipulate that charges couple to fields in such a way that their conservation laws are dynamically respected. But Gauss's law can clearly fluster cluster decomposition: once one knows the electric flux around a closed surface, one knows precisely the amount of charge within it. Quantities measured on the totality of the boundary are therefore not independent of other quantities measured in the bulk. Of course, this type of "synchronic non-locality" is not causal; it only represents "non-locally possessed" properties (in the language of Belot (1998) and Healey (2007)). That is, there should be room for holism, which this paper has drawn on.

The work of Gomes and Riello (2019) reported here precisely delineates the sort of non-locality involved. For generic regional states in Yang-Mills theory, one does not have $\neg$ GSS: in most circumstances we can describe the physical state of the whole by describing the physical state of its composing regions. When the regional states do not uniquely determine the universal state, $\neg$ GSS ensues. In the Abelian case, this $\neg$ GSS maps onto DES as I have defined it. That is, it is a particular non-locality that is responsible for the gap between the regional gauge-invariant information and the universal gauge-invariant information; it is this gap from which DES emerges.

In the non-Abelian case, a boundary can have stabilizers which are not shared by the bulk of the regions. These will also result in $\neg$ GSS, but, as argued in appendix $\mathrm{A}$, this variety cannot be associated with DES, as it has no unique representation as a transformation of the regional states.

Moreover, as expected, the group of symmetries with DES has intimate connections with the charge group of the theory: For both Abelian and non-Abelian theories the relevant residual variety of universal physical states can be parametrized by subalgebras of $\operatorname{Lie}(G)$. And these rigid symmetries with DES are in 1-1 correspondence with the possible (covariantly) conserved charges in the region (cf. (Gomes \& Riello, 2019, Sec. 4.3.2)). In the Galileo's ship example, the variety is given by the action of the Galilean group, 'Boosts $\ltimes$ Euclidean,' on the subsystem, and the respective charges are the conserved momenta. 
In a few words: for the physical, i.e. gauge-invariant, content of the gauge fields, the whole can be more than the sum of the parts. Their difference manifests itself in a direct empirical significance of (subgroups of) the charge group, and are in 1-1 relation to the conserved charges of the theory. The same can be said for Galileo's ship scenario, where the difference manifests itself in the direct empirical significance of the Galilean group.

\subsection{The role of the subsystem}

The conclusion we have arrived at says that DES are not inextricable from other effects of symmetries. In particular, I have related direct empirical significance (DES) to a failure of supervenience on subsystems ( $\neg \mathrm{GSS}$ ), and then stated that that sort of $\neg \mathrm{GSS}$ is related to the indirect significance of symmetry (labeled IES), like conservation of charges.

Given the sort of holism we have seen in $\neg$ GSS, it thus might be conceptually preferable to use global information about charges and other superselected quantities pertaining to IES to parametrize the possible gluings of regions in a gauge-free manner. In that case, we may not want to invoke external sophistication.

Certainly in practice we will often lack the relevant global information, and so it makes sense to use a local gauge-fixed parametrization to do calculations, make predictions, etc. Similarly, insofar as we only have a perturbative handle on the gluing procedure in the non-Abelian case, and our current perturbation theory relies on compactly localized fields, there is reason to use gauge-fixed compactly-localized fields instead of gauge-invariant non-compactly localized fields, and external sophistication gives a conceptual underpinning to the ensuing gluing procedures. But these sorts of concerns are probably best seen as practical or technical barriers, not as in-principle reasons to adopt external sophistication.

Indeed, from a global perspective external sophistication loses its warrant. But then, so does the empirical relation between conserved charges and rigid symmetries, since DES necessarily involves an external perspective.

\subsection{Summary}

Broadly speaking, in this paper I have explored the role of $\neg$ GSS in the context of gauge theories. I dissected the meaning and occurrence of "empirically significant subsystem symmetries", whose existence and characteristics are still matters of debate in the philosophy of physics. To make matters concrete, I have focused on Yang-Mills theory (and, in more detail, electromagnetism) and restricted subsystems to be demarcated by spacetime regions.

In order to better adjudicate the debate between the opposing sides-represented by Greaves and Wallace (2014) on one side, and Brading and Brown (2004) on the other- it was first necessary to clear the ground by introducing new nomenclature. The standard nomenclature of local and global gauge symmetries is perfectly adequate if the system under study is the entire Universe, as is usually the case. But if one wants to discuss subsystems, and needs to distinguish between symmetries acting 
also at these different levels, the standard nomenclature becomes awkward. The awkwardness is apparent when one refers, e.g.: to 'a global subsystem symmetry'. The new nomenclature uses four labels to express two logically independent distinctions: namely (i) whether symmetries act only regionally or universally, and (ii) whether they depend on an infinite or a finite set of parameters, i.e. whether they are rigid or malleable when acting throughout a spacetime region or throughout the universe. It also disentangles possible confusions with 'non-local and local' functions.

With these definitions in place, and in the specific setting of Yang-Mills theory in bounded regions, I found myself in agreement with Greaves and Wallace (2014) in their criticism of Brading and Brown (2004). Namely, Brading and Brown (2004) prematurely dismissed the possibility of DES tranformations by assuming that such a transformation would always create discontinuities in the boundary between the regions. The difference between Greaves and Wallace (2014) and Brading and Brown (2004) can be briefly summarized thus: one should be concerned with smooth composition of regional gauge and matter fields (Greaves \& Wallace, 2014), as opposed to a smooth composition of gauge transformations (Brading \& Brown, 2004), which are not physical.

But the gluing of the gauge and matter fields is also not physical; only the composition of their gauge equivalence classes is. This misunderstanding is reflected in Greaves and Wallace (2014)'s explicit assumption of supervenience on regions (cf. GSS' in Section 4.3), that is, that regional states should uniquely define the universal state. Greaves and Wallace (2014) admit this condition fails for gauge theories in certain gauge-invariant bases (holonomies for the Abelian case), but it even clearly fails for Galileo's ship. Thus GSS seems inconsistent with an appropriate definition of DES for gauge theories.

My interpretation of Greaves and Wallace (2014) and Brading and Brown (2004)'s oversight is that they forget aspects of the Definition 1 of DES: they do not check whether condition 1 is satisfied explicitly (by employing gauge-invariance information) and they do not consider condition 2 as involving physical information that is intrinsic to a subsystem or region.

Having noticed these issues, we could summarize the journey from the present results to Greaves and Wallace (2014) and from there to Brading and Brown (2004) as follows: one should be concerned with the smooth composition of the physical data, as given by gauge-equivalence classes, and not with smooth composition of gauge and matter fields through given representatives (Greaves \& Wallace, 2014), and much less with the smooth composition of gauge transformations (Brading \& Brown, 2004).

With a clear course set, we were ready to provide a more precise definition of DES. Finding the criteria for smooth composition of the information contained in the equivalence classes $\left[\varphi_{ \pm}\right]$still presented a challenge. The challenge was: can their physical content be composed into a universal physically valid state?

The way we got around the problem of gluing physical content was to use an 'externally sophisticated view of symmetries' ${ }^{44}$ Namely, I employed 'reduction',

\footnotetext{
${ }^{44}$ Such a view is intimately related to composition, as advocated by Rovelli (2014); see also Gomes (2019) for the same issue in a context of regional subsystems.
} 
or better, 'projection', as a means to identify the regional and universal physical contents-each non-locally determined within its corresponding domain,- - and then I employed sophistication for gluing.

We found that for electromagnetism as coupled to a scalar Klein-Gordon fieldwhen the Klein Gordon fields were taken to vanish at the boundary in between the regions, but not in the bulk within them-there can be multiple universal physical states formed by gluing the same regional physical states. That is, a failure of uniqueness in the gluing creates the gap which gives a residual variety of universal physical states. In other words, a variety of universal physical states can be built from the same regional physical states. These regional and universal states fulfill our Definition 2 for $\neg \mathrm{GSS}$, and thereby also correspond to purely relational DES, as described in Definition 1.

Even though I have used 'external sophistication', Theorem 1, including its significance for DES, is completely compatible with viewing gauge degrees of freedom as "descriptive fluff". The fact that the theory admits such a particular sort of redundancy is related to the particular sort of non-locality of its gauge-independent degrees of freedom.

In the landscape of the debate, we thus locate ourselves somewhere between the two opposing views. On the side of Greaves and Wallace (2014), we find that gauge theories may indeed harbor symmetries that have direct empirical significance. On the side of Brading and Brown (2004) and the orthodoxy, ${ }^{45}$ we find that indeed only rigid symmetries may have direct empirical significance in gauge theory. In fact, surprisingly, the rigid ones correspond to 'the global' gauge transformations, $G$ (for non-Abelian: only for very particular perturbed configurations, that can carry associated covariantly conserved charges). But to force my conclusion into either pigeonhole, or even a combination, would be to shave off some of the important subtleties of this situation.

Acknowledgements I would like to thank Aldo Riello, my collaborator in all things gauge-related, for many fruitful and insightful discussions and for co-developing the entire technical apparatus employed in this analysis. And similarly, I would like to thank Jeremy Butterfield, for many discussions, for many requests for clarification, for patiently guiding me in all things philosophy-related, and for giving immensely valuable and thorough feedback on this paper.

\section{Appendix A. Non-Abelian Yang-Mills}

\section{A. 1 Notation for Yang-Mills theory coupled to matter}

The general case works with a finite-dimensional charge group, e.g. $G=\operatorname{SU}(N)$, with Lie algebra $\mathfrak{g}:=\operatorname{Lie}(G)$, e.g. $\mathfrak{g}=\mathfrak{s u}(N)$. Given the charge group we define the group of gauge transformations $\mathcal{G}=C^{\infty}(\Sigma, G)$, with composition given by pointwise action of $G$, i.e. $\left(g g^{\prime}\right)(x):=g(x) g^{\prime}(x)$, and the respective infinitesimal

\footnotetext{
${ }^{45}$ In fact, in this point, we roughtly align with Kosso (2000) - but not always: only when covariantly conserved charges exist-and not with Brown and Brading, who want to claim that even rigid internal symmetries have no DES.
} 
version, $\operatorname{Lie}(\mathcal{G})=C^{\infty}(\Sigma, \mathfrak{g})$ (with pointwise Lie algebra commutator). An element of $\mathcal{G}$ is a map: $g(\cdot): \Sigma \ni x \mapsto g(x) \in G$. The gauge fields and its gauge-transformed version $A^{g}$ are given by Lie-algebra valued space(time) 1-forms.

We define, for $\mathrm{x}^{\mu}$ a basis of 1 -forms over $\Sigma$ and $\tau_{\alpha}$ a basis of the Lie algebra $\mathfrak{g}$

$$
A=A_{\mu}^{\alpha} \mathrm{x}^{\mu} \tau_{\alpha} \in \Lambda^{1}(\Sigma, \mathfrak{g}) \quad \text { and } \quad A^{g}:=g^{-1} A g+g^{-1} \partial g
$$

Infinitesimally, i.e. for an infinitesimal gauge-transformation $\xi \in \operatorname{Lie}(\mathcal{G})$, the gauge field transforms as

$$
A \mapsto A+\mathrm{D} \xi \quad \text { where } \quad \mathrm{D} \xi:=\partial \xi+[A, \xi] .
$$

For full generality we can introduce charged fermions in a fundamental representation: so for some vector space $W$, the 4 -component Dirac spinor field (i.e. in $\mathbb{C}^{4}$ ) as:

$$
\psi \in C^{\infty}\left(\Sigma, \mathbb{C}^{4} \otimes W\right) \text { and } \psi^{g}=g \psi .
$$

And I will write the joint configuration as

$$
\varphi=(A, \psi)
$$

I will denote the regional, unquotiented configuration spaces of each field sector (gauge field and matter, respectively) as $\mathcal{A}_{ \pm}=\left\{A_{ \pm} \in \Lambda^{1}\left(\Sigma_{ \pm}, \operatorname{Lie}(G)\right)\right\}$, $\Psi_{ \pm}=\left\{\psi_{ \pm} \in C^{\infty}\left(\Sigma_{ \pm}, \mathbb{C}^{4} \otimes W\right)\right\}$. And, for the joint configuration spaces, I write $\Phi_{ \pm}=\left\{\varphi_{ \pm}:=\left(A_{ \pm}, \psi_{ \pm}\right) \in \mathcal{A}_{ \pm} \times \Psi_{ \pm}\right\}$, writing $\Phi, \Psi, \mathcal{A}$. I will omit the subscript \pm for the corresponding universal configuration spaces. The restricted groups of gauge transformations will be denoted in analogous fashion: $\mathcal{G}_{ \pm}=C^{\infty}\left(\Sigma_{ \pm}, G\right)$, and all abstract quotient spaces are denoted by the square brackets, as in $\left[\Phi^{ \pm}\right]:=\Phi^{ \pm} / \mathcal{G}_{ \pm}$, and $[\Phi]:=\Phi / \mathcal{G}$.

\section{A.2 Gluing for non-Abelian Yang-Mills theory}

In the non-Abelian case we have the equations analogous to Eq. 3.8 and Eq. 3.12, namely:

$$
\begin{gathered}
\left\{\begin{array}{c}
\mathrm{D}^{i} \delta A_{i}^{ \pm}=0 \\
s^{i} \delta A_{i}^{ \pm} \mid S=0
\end{array}\right. \\
\mathrm{D}^{i} \delta A_{i}=0
\end{gathered}
$$

And we apply them to establish gluing, by first writing:

$$
\delta A:=\left(\delta A^{++} \mathrm{D} \xi^{+}\right) \Theta_{+}+\left(\delta A^{-}+\mathrm{D} \xi^{-}\right) \Theta_{-} .
$$

We obtain the following conditions on $\xi_{ \pm}$:

$$
\left\{\begin{array}{l}
\mathrm{D}^{2} \xi_{ \pm}=0 \quad \text { on } \quad \Sigma_{ \pm} \\
s_{i} \mathrm{D}^{i}\left(\xi_{+}-\xi_{-}\right)=0 \quad \text { on } \quad S \\
\left(\xi_{+}-\xi_{-}\right)_{\mid S}=\left(\mathrm{D}_{S}^{2}\right)^{-1}\left(\mathrm{D}_{S}^{c}\left(\delta A_{c}^{+}-\delta A_{c}^{-}\right)_{\mid S}\right) \quad \text { on } \quad S
\end{array}\right.
$$

(subscript $S$ means intrinsic to the surface). 
We can solve these emerging conditions (see Section 4 in Gomes and Riello (2019)), obtaining:

$$
\xi^{ \pm}=\zeta_{( \pm)}^{ \pm \Pi} \quad \text { with } \quad \Pi=\left(\mathcal{R}_{+}^{-1}+\mathcal{R}_{-}^{-1}\right)^{-1}\left(\left(\mathrm{D}_{S}^{2}\right)^{-1} \mathrm{D}_{S}^{c}\left(\delta A_{c}^{+}-\delta A_{c}^{-}\right)_{\mid S}\right)
$$

where, in each region, $\zeta^{\phi}$ stands for a covariantly harmonic function, satisfying $\mathrm{D}^{2} \zeta=0$, with Neumann boundary conditions at $S$ given by $s^{i} \mathrm{D}_{i} \zeta \mid S=\phi$, and where the subscript $S$ denotes "intrinsic to $S$ ", and the intrinsic coordinates to $S$ are given by $c$, and where $\mathcal{R}$ is the so-called Dirichlet-to-Neumman operator. Briefly, $\mathcal{R}$ functions as follows: a given harmonic function with Dirichlet conditions-these conditions are the input of $\mathcal{R}$-will possess a certain normal derivative at the boundary; i.e. will induce certain Neumann conditions there-these conditions are the output of $\mathcal{R}$. That is, let $\zeta^{u}$ be a harmonic function with Neumann boundary condition, then for $\zeta_{u}$ a covariantly harmonic function with Dirichlet boundary condition, $\left(\zeta_{u}\right)_{\mid S}=u$, the Dirichlet to Neumann operator $\mathcal{R}$ is defined as $\zeta_{u}=\zeta^{\mathcal{R}(u)}$ (i.e. it finds the harmonic function with Neumann condition corresponding to one with a Dirichlet condition).

In words here is what the theorem means. In a given region, say $\Sigma_{+}$, with respect to the covariant differential operator $\mathrm{D}^{+}$, the vertical $\xi_{+}$which translates between the global and regional horizontals, $H_{\mid R_{+}}=h_{+}+\mathrm{D} \xi_{+}$, is defined as a harmonic function with Neumann boundary conditions. The Neumann conditions are implicitly defined by the difference of horizontals at the boundary, but since this difference would only give a Dirichlet boundary condition, one must apply the Dirichlet to Neumann boundary operator. Nonetheless, we can summarize: $\xi_{ \pm}$are the unique harmonic functions with Neumann conditions defined by the difference of horizontals at the boundary. Each such doublet will identify a unique global physical state compatible with the doublet of horizontals, $\left(h_{+}, h_{-}\right)$.

\section{A.3 Non-Abelian $\neg$ GSS}

There are two sources of under-determination of solutions to Eq. A.5: one topological and one from homogeneous fields. The first arises from the possibly non-trivial first (equivariant) homology group of $\Sigma$, we look at the first in Section A.3.1 and at the second in Section A.3.2.

\section{A.3.1 Topological holism}

We may have a universal field $\delta A$ satifying Eq. A.4 which, when restricted to each $\Sigma_{ \pm}$is pure gauge, i.e. of the form $\mathrm{D} \xi_{ \pm}$. In other words, the room for discrepancy is equivalent to broken homology cycles. This is easier to see in the Abelian case, where $\delta A$ would be a one form which is exact in the simply connected patches, but only closed in the entire manifold (see Gomes and Riello (2019), Section 4.7 for an example). By the Poincaré lemma, this occurs if and only if the topology of the manifold is non-trivial. In other words, universal physical processes in this case may not come from regional physical processes; some physical processes are universal/global in nature. 
But of course for topologically non-trivial manifolds we should expect the whole to contain more information than the sum of the parts, foiling GSS. The arising $\neg$ GSS would contribute with the suitable homology group to the variety set $I$ in Eq. 2.2. Such a contribution has a finite number of generators (given by the first Betti number, i.e. the rank of the first homology group). ${ }^{46}$

\section{A.3.2 Stabilizer ambiguity}

Barring non-trivial topology, is there another source of under-determination of $\xi^{ \pm}$ also in the non-Abelian case? To arrive at an answer, note that we are using the gauge-fields as a reference. It is through them that we fix the $\xi$ and parametrize the reduced/quotient configuration space $[\Phi]=\Phi / \mathcal{G}$. Therefore one source of $\neg$ GSS would be a possible under-determination of $\xi_{ \pm}$.

This under-determination occurs if and only if there exists a boundary transformation $\chi_{S} \in \operatorname{Lie}(\mathcal{G})_{S}$ such that

$$
{ }^{S} \mathrm{D} \chi_{S}=0
$$

That is, if there exist boundary stabilizers.

In most cases these stabilizers need not be prolongated into the regions. However, the special case in which $\chi_{S}=\chi^{+}-\chi_{-}$for regional stabilizers,

$$
\mathrm{D} \chi_{ \pm}=\partial \chi_{ \pm}+\left[A_{ \pm}, \chi_{ \pm}\right]=0
$$

for $A=A_{+} \Theta_{+}+A_{-} \Theta_{-}$being the smooth field configuration around which we are considering perturbations, we get DES in the sense of the main text. This is, again, the "Killing" (or infinitesimal stabilizer) equation for gauge transformations. Namely, under-determination of $\xi_{ \pm}$occurs if and only if there exist $\chi_{S}$ which stabilize the boundary value of $A_{S}$. The effect this ambiguity has on the $\xi_{ \pm}$is gauge-fixing dependent, and arises from an ambiguity in $\Pi$ in Eq. A.6, of the form $\Pi \rightarrow \Pi+$ $\left.\mathcal{R}_{-}^{-1}\right)^{-1}\left(\chi_{S}\right)$.

However, if $\chi_{S}=\left(\chi_{+}-\chi_{-}\right)_{\mid S}$ where $\chi_{ \pm}$stabilize the entire $A_{ \pm}$, this ambiguity becomes much simpler to express: we can use $\xi_{ \pm}$or $\xi_{ \pm}+\chi_{ \pm}$for gluing. But in this latter case, only if there is matter will the difference come with an effect in the representation of the glued states. That is, the effect of a change $\xi_{ \pm} \rightarrow \xi_{ \pm}+\chi_{ \pm}$ is trivial without matter, since $\mathrm{D}_{ \pm} \chi_{ \pm}=0$. Such stabilizers are generalizations of the constant potential shifts in electromagnetism. Here the entire regional field must be stabilized by $\chi_{ \pm}$, and this singles out, from $\mathcal{G}^{ \pm}$, the regional elements which can potentially exhibit DES.

As with Killing directions for spacetime geometries, such elements are hard to come by: they're generically trivial (for non-Abelian charge groups $G$ ), and, when non-trivial, they are generated by a finite-dimensional basis.

If the boundary stabilizers are inextendible to the regions, then each will correspond with a different regional gauge-fixed configuration, and thus it will result in

\footnotetext{
${ }^{46}$ This topological fact should be consequential to the labeling of the inequivalent representations of the $\theta$ vacua (see Strocchi (2015)).
} 
physical variety. But this difference is not attributed to a regional rigid symmetry and thus cannot be associated with DES.

In sum: there may still be some small variety in $\xi^{ \pm}$, which is innocuous as far as the gauge field is concerned, and some variety that is relevant. The former variety cannot be uniquely associated with a regional transformation, whereas, as we will shortly see, the latter variety can be uniquely associated with a rigid regional transformation with DES, but it can only be relationally felt by regional matter fields.

From now on we will ignore those transformation that only stabilize the boundary values of the field, and focus on the ones that are intersting in the context of DES.

\section{A.3.3 Obtaining variety from the regional stabilizer ambiguity}

When we include matter fields, the universal field is composed as:

$$
\delta \psi=\left(\delta \psi^{++} \xi^{+} \psi_{+}\right) \Theta_{+}+\left(\delta \psi^{-}+\xi^{-} \psi\right)
$$

Since we are not using the matter fields to parametrize the quotient/moduli/reduced configuration spaces, there are no analogues of equations (3.8) and (3.12) to be implemented for it. But smoothness of the field does impose certain conditions. In fact, different choices of stabilizer, adding $\chi_{+} \neq \chi_{-}$to $\xi_{+}, \xi_{-}$, respectively, will render the matter fields incompatible at the boundary. ${ }^{47}$

Therefore, if the matter fields do not vanish at the boundary, we can assume there is again regional determinacy as per Definition 2. So we assume they vanish there; a condition postulated by Greaves and Wallace (2014) as "dynamical isolation" of the two regions. And also, if the matter field vanishes not only at the boundary, but everywhere, no $\neg$ GSS, i.e. GSS arises, yet again. Thus, I assume: the matter fields vanish at the boundary, but not in the bulk of the regions.

To illustrate the precise emergence of the Lie algebra $\mathfrak{g}$, we resort to a 'best-case' scenario. That is, apart from the conditions stipulated above for the matter fields, we will take the gauge field configuration around which we are perturbing to be the 'vacuum', $A=0$. In that case, the gauge covariant differential just becomes the standard differential (as in the Abelian, electromagnetic case), i.e. $\mathrm{D} \rightarrow \partial$, and the stabilizer equation becomes $\left(\partial \chi^{\alpha}(x)\right) \tau_{\alpha}=0$. Then not only is the space of stabilizers $\chi$ a finite-dimensional vector space, closed under commutation, but for this case it forms a Lie algebra homomorphic to $\mathfrak{g}$.

Then we have a parametrized solution

$$
\delta \psi_{\left(\chi^{+}, \chi^{-}\right)}=\left(\delta \psi^{++}\left(\xi^{++} \chi^{+}\right) \psi_{+}\right) \Theta_{+}+\left(\delta \psi^{-}+\left(\xi^{-}+\chi^{-}\right) \psi\right)
$$

But we are still not done. For $\chi^{+}=\chi^{-}, \delta \psi$ is still universally gauge equivalent to $\delta \psi_{\left(\chi^{+}, \chi^{-}\right)}$, i.e. they differ by a universal, rigid gauge transformation. Therefore, what really matters for universal variety is the difference: $\chi_{+}-\chi_{-}$. This difference

\footnotetext{
47 That is, unless $\chi^{ \pm}$also stabilize $\psi^{ \pm}$. For non-Abelian fields, there may still be internal stabilized directions, i.e. for particular configurations $\tilde{\psi}_{ \pm} \neq 0$, there may be $\tilde{\chi}_{ \pm} \neq 0$ such that $\tilde{\chi}_{ \pm} \psi_{ \pm}=0$. This is not true for $\mathrm{U}(1)$, there $\tilde{\chi}_{ \pm} \psi_{ \pm} \neq 0$ for any non-vanishing gauge transformation and matter field. But I do not know of any non-trivial, shared stabilizers for both fields in such a situation.
} 
is generated by just one copy of $\mathfrak{g}$. That is, given the $\delta A_{ \pm}, \delta \psi_{ \pm}$, we have:

$$
\left(\delta A, \delta \psi_{(i)}\right)=\left(\delta A^{++} \mathrm{D} \xi_{(i)}^{+}, \delta \psi^{++} \xi_{(i)}^{+} \psi^{+}\right) \Theta_{+}+\left(\delta A^{-}+\mathrm{D} \xi^{-}, \delta \psi^{-}+\xi^{-} \psi^{-}\right) \Theta_{-}
$$

where $\xi_{(i)}^{+}(x):=\left(\xi_{\alpha}^{+}(x)+i_{\alpha}\right) \tau^{\alpha}$, for spacetime constant coefficients $i^{\alpha}$, with $\tau_{\alpha}$ a basis of $\mathfrak{g}$ (which also implies $\mathrm{D} \xi^{+}=\mathrm{D} \xi_{(i)}^{+}$), i.e. the $i$ parametrize the Lie algebra of the charge group $G$.

Finally, we have arrived at our destination: I have shown that the variety set $I$, at least infinitesimally and in the best-case scenario, can in some sense recover the charge group $G$, i.e. I have characterized the infinitesimal version of Eq. 3.3, with:

$$
\left[\delta \varphi^{(i)}\right]=\left[\delta \varphi^{+}\right] \cup_{(i)}^{S}\left[\delta \varphi^{-}\right], \quad i \in \mathfrak{g}, \quad \text { with } \quad\left[\delta \varphi^{(i)}\right] \neq\left[\delta \varphi^{\left(i^{\prime}\right)}\right] \quad \text { iff } \quad i \neq i^{\prime}
$$

For non-Abelian groups, it is not clear how to obtain the finite version of this equation, replacing $\delta \varphi$ with $\varphi$, i.e. with

$$
\left[\varphi^{(i)}\right]=\left[\varphi^{+}\right] \cup_{(i)}^{S}\left[\varphi^{-}\right], \quad i \in I \simeq G
$$

But in the Abelian case, the tools utilized here do allow an integration to the finite setting. 48

Moving away from the best-case scenario of $A=0$ : to obtain $\neg$ GSS the base configuration $A$ must still be somewhat homogenous; it must admit internal directions which leave it unchanged. But as mentioned above, a general theorem about Killing fields (and stabilizers), shows that Killing directions are always generated by a finite dimensional basis; in the case of internal gauge transformations, this basis consists of at $\operatorname{most} \operatorname{dim}(\mathfrak{g})$ elements, closed under commutation, etc. Thus even away from the best-case, we obtain I's isomorphic to sub-algebras of $\mathfrak{g}$ (we would also have to replace $\tau_{\alpha}$ in the definition of $\delta \psi_{(i)}$ for a choice of basis $\left\{\chi_{\alpha}\right\}$ of the appropriate Killing, or stabilizing fields).

Note that, as had to be the case, according to Definition 2, there exists a regional variety implicit in Eq. A.11, and this variety recovers also the definition of DES through transformations, if the transformations referred to in Definition 1 are taken to be e.g.: $(\delta A, \delta \psi) \rightarrow\left(\delta A, \delta \psi_{(i)}\right)$.

Open Access This article is licensed under a Creative Commons Attribution 4.0 International License, which permits use, sharing, adaptation, distribution and reproduction in any medium or format, as long as you give appropriate credit to the original author(s) and the source, provide a link to the Creative Commons licence, and indicate if changes were made. The images or other third party material in this article are included in the article's Creative Commons licence, unless indicated otherwise in a credit line to the material. If material is not included in the article's Creative Commons licence and your intended use is not permitted by statutory regulation or exceeds the permitted use, you will need to obtain permission directly from the copyright holder. To view a copy of this licence, visit http://creativecommons.org/licenses/by/4.0/.

\footnotetext{
${ }^{48}$ This is related to the 'dressing formalism", see Section 9 of Gomes et al. (2019) and François (2019) and Attard et al. (2018).
} 


\section{References}

Attard, J., François, J., Lazzarini, S., \& Masson, T. (2018). The dressing field method of gauge symmetry reduction, a review with examples. In J. Kouneiher (Ed.) Foundations of mathematics and physics one century after hilbert: New perspectives (pp. 377-415). Cham: Springer International Publishing. https://doi.org/10.1007/978-3-319-64813-2_13.

Balachandran, A. P., Chandar, L., \& Momen, A. (1996). Edge states in gravity and black hole physics. Nucl. Phys., B461, 581-596.

Barnich, G., \& Brandt, F. (2002). Covariant theory of asymptotic symmetries, conservation laws and central charges. Nucl. Phys., B633, 3-82.

Barrett, J. W. (1991). Holonomy and path structures in general relativity and Yang-Mills theory. International Journal of Theoretical Physics, 30(9), 1171-1215. https://doi.org/10.1007/BF00671007.

Belot, G. (1998). Understanding electromagnetism. The British Journal for the Philosophy of Science, 49(4), 531-555. https://doi.org/10.1093/bjps/49.4.531.

Brading, K., \& Brown, H. R. (2000). Noether's theorems and gauge symmetries.

Brading, K., \& Brown, H. R. (2004). Are Gauge Symmetry Transformations Observable? The British Journal for the Philosophy of Science, 55(4), 645-665. http://www.jstor.org/stable/3541620.

Brading, K., \& Castellani, E. (Eds.) (2003). Symmetries in Physics: Philosophical Reflections. Cambridge: Cambridge University Press.

Butterfield, J. (2007). On symplectic reduction in classical mechanics. In J. Butterfield, \& J. Earman (Eds.) Philosophy of Physics (pp. 1-131). Amsterdam: North-Holland. http://www.sciencedirect. com/science/article/pii/B978044451560550004X.

Caulton, A. (2015). The role of symmetry in the interpretation of physical theories. Studies in History and Philosophy of Science Part B: Studies in History and Philosophy of Modern Physics, 52, 153-162. https://doi.org/10.1016/j.shpsb.2015.08.002.

Crnkovic, C., \& Witten, E. (1987). Covariant description of canonical formalism in geometrical theories. In S. W. Hawking, \& W. Israel (Eds.) Three hundred years of gravitation (pp. 676-684). Cambridge.

Dewar, N. (2017). Sophistication about symmetries. The British Journal for the Philosophy of Science, 70(2), 485-521. https://doi.org/10.1093/bjps/axx021.

Dirac, P. A. M. (1955). Gauge invariant formulation of quantum electrodynamics. Can. J. Phys., 33, 650.

Donnelly, W., \& Freidel, L. (2016). Local subsystems in gauge theory and gravity. JHEP, 09, 102.

Dougherty, J. (2017). Sameness and separability in gauge theories. Philosophy of Science, 84(5), 11891201. https://doi.org/10.1086/694083.

Earman, J. (1987). Locality, nonlocality and action at a distance: a skeptical review of some philosophical dogmas. In R. Kargon, P. Achinstein, \& W. T. Kelvin (Eds.) Kelvin's baltimore lectures and modern theoretical physics : historical and philosophical perspectives (pp. 449-490). Cambridge: MIT Press. http://d-scholarship.pitt.edu/12972/.

Earman, J. (2019). The role of idealizations in the Aharonov-Bohm effect. Synthese, 196(5), 1991-2019. https://doi.org/10.1007/s11229-017-1522-9.

Fischer, A. (1970). The theory of superspace. In M. Carmeli, S. I. Fickler, \& L. Witten (Eds.) Proceedings of the relativity conference held 2-6 June, 1969 in Cincinnati, OH (p. 303). New York: Plenum Press.

Fletcher, S. (2021). An invitation to approximate symmetry, with three applications to intertheoretic relations. Synthese, 198(5), 4811-4831. Publisher Copyright: @2019, Springer Nature B.V.

François, J. (2019). Artificial versus substantial gauge symmetries: a criterion and an application to the electroweak model. Philosophy of Science, 86(3), 472-496. https://doi.org/10.1086/703571.

Giulini, D. (1995). Asymptotic symmetry groups of long-ranged gauge configurations. Modern Physics Letters A, 10(28), 2059-2070. https://doi.org/10.1142/S0217732395002210.

Gomes, H. (2019). Gauging the boundary in field-space. Studies in History and Philosophy of Science Part B: Studies in History and Philosophy of Modern Physics. http://www.sciencedirect.com/science/ article/pii/S1355219818302144.

Gomes, H. (2020). Gauge-invariance and the direct empirical significance of symmetries. (to appear in Studies in History and Philosophy of Modern Physics).

Gomes, H., Hopfmüller, F., \& Riello, A. (2019). A unified geometric framework for boundary charges and dressings: Non-Abelian theory and matter. Nuclear Physics B, 941, 249-315. http://www. sciencedirect.com/science/article/pii/S0550321319300483. 
Gomes, H., \& Riello, A. (2017). The observer's ghost: notes on a field space connection. Journal of High Energy Physics (JHEP), 05, 017. https://link.springer.com/article/10.1007\%2FJHEP05 $\% 282017 \% 29017$.

Gomes, H., \& Riello, A. (2019). Quasilocal degrees of freedom in Yang-Mills theory. Forthcoming in SciPost: https://scipost.org/submissions/scipost_202001_00038v1/.

Greaves, H., \& Wallace, D. (2014). Empirical consequences of symmetries. British Journal for the Philosophy of Science, 65(1), 59-89.

Healey, R. (2007). Gauging What's real: the conceptual foundations of gauge theories. Oxford: Oxford University Press.

Healey, R. (2009). Perfect symmetries. The British Journal for the Philosophy of Science, 60(4), 697-720. https://doi.org/10.1093/bjps/axp033.

Henneaux, M., \& Teitelboim, C. (1992). Quantization of gauge systems. Princeton: Princeton University Press.

'T Hooft, G. (1980). Gauge theories and the forces between elementary particles. Scientific American, $242,90-166$.

Kobayashi, S., \& Nomizu, K. (1963). Foundations of differential geometry. Vol I. Interscience Publishers, a division of John Wiley \& Sons, New York-London.

Kondracki, W., \& Rogulski, J. (1983). On the stratification of the orbit space for the action of automorphisms on connections. On conjugacy classes of closed subgroups. On the notion of stratification. Inst., Acad. https://books.google.co.uk/books?id=LK0JrgEACAAJ.

Kosmann-Schwarzbach, Y., \& Schwarzbach, B. E. (2011). The noether theorems: Invariance and conservation laws in the twentieth century. New York: Springer.

Kosso, P. (2000). The empirical status of symmetries in physics. The British Journal for the Philosophy of Science, 51(1), 81-98. http://www.jstor.org/stable/3541749.

Ladyman, J. (2015). Representation and symmetry in physics. unpublished.

Lee, J., \& Wald, R. M. (1990). Local symmetries and constraints. J. Math. Phys., 31, 725-743.

Maudlin, T. (1993). Buckets of water and waves of space: why spacetime is probably a substance. Philosophy of Science, 60(2), 183-203. http://www.jstor.org/stable/188350.

Maudlin, T. (1998). Healey on the Aharonov-Bohm effect. Philosophy of Science, 65(2), 361-368. http:// www.jstor.org/stable/188266.

Myrvold, W. C. (2010). Nonseparability, classical, and quantum. The British Journal for the Philosophy of Science, 62(2), 417-432. https://doi.org/10.1093/bjps/axq036.

Nguyen, J., Teh, N. J., \& Wells, L. (2018). Why surplus structure is not superfluous. The British Journal for the Philosophy of Science. https://doi.org/10.1093/bjps/axy026.

Noether, E. (1917). Invariant variation problems. Transport Theory and Statistical Physics, 1(3), $186-207$. https://doi.org/10.1080/00411457108231446.

Pitts, J. B. (2011). Permanent underdetermination from approximate empirical equivalence in field theory: Massless and massive scalar gravity, neutrino, electromagnetic, Yang-Mills and gravitational theories. The British Journal for the Philosophy of Science, 62(2), 259-299. https://doi.org/10.1093/bjps/axq014.

Pooley, O. (2013). Substantivalist and relationalist approaches to spacetime, (chap. 15). In R. Batterman (Ed.) The Oxford Handbook of Philosophy of Physics. Oxford University Press.

Ramirez, S., \& Teh, N. (2019). Abandoning Galileo's ship: the quest for non-relational empirical signicance. preprint.

Regge, T., \& Teitelboim, C. (1974). Role of surface integrals in the hamiltonian formulation of general relativity. Annals Phys., 88, 286.

Riello, A. (2019). Soft charges from the geometry of field space. JHEP.

Rovelli, C. (2014). Why gauge? Foundations of Physics, 44(1), 91-104.

Strocchi, F. (2015). Symmetry breaking, gauge symmetries.

Strominger, A. (2018). Lectures on the infrared structure of gravity and gauge theory. Princeton: Princeton University Press.

Teh, N. J. (2016). Galileo's gauge: Understanding the empirical significance of gauge symmetry. Philosophy of Science, 83(1), 93-118. https://doi.org/10.1086/684196.

Publisher's note Springer Nature remains neutral with regard to jurisdictional claims in published maps and institutional affiliations. 